\title{
The influence of the cell inclination on the heat transport and large-scale circulation in liquid metal convection
}

\author{
Lukas Zwirner ${ }^{1, \dagger}$, Ruslan Khalilov ${ }^{2}$, Ilya Kolesnichenko ${ }^{2}$, Andrey Mamykin ${ }^{2}$, \\ Sergei Mandrykin ${ }^{2}$, Alexander Pavlinov ${ }^{2}$, Alexander Shestakov ${ }^{2}$, \\ Andrei Teimurazov ${ }^{2}$, Peter Frick ${ }^{2}$ and Olga Shishkina ${ }^{1}$ \\ ${ }^{1}$ Max Planck Institute for Dynamics and Self-Organization, Am Fassberg 17, 37077 Göttingen, Germany \\ ${ }^{2}$ Institute of Continuous Media Mechanics, Korolyov 1, Perm 614013, Russia
}

(Received 4 April 2019; revised 25 October 2019; accepted 5 November 2019)

Inclined turbulent thermal convection in liquid sodium is studied at large Rayleigh numbers $R a \gtrsim 10^{7}$ based on the results of both experimental measurements and high-resolution numerical simulations. For a direct comparison, the considered system parameters are set to be similar: $R a=1.67 \times 10^{7}$ in the direct numerical simulations (DNS), $R a=1.5 \times 10^{7}$ in the large-eddy simulations and $R a=1.42 \times 10^{7}$ in the experiments, while the Prandtl number of liquid sodium is very small $(\operatorname{Pr} \approx 0.009)$. The cylindrical convection cell has an aspect ratio of one; one circular surface is heated, while the other one is cooled. Additionally, the cylinder is inclined with respect to gravity and the inclination angle varies from $\beta=0^{\circ}$, which corresponds to Rayleigh-Bénard convection (RBC), to $\beta=90^{\circ}$, as in a vertical convection (VC) set-up. Our study demonstrates quantitative agreement of the experimental and numerical results, in particular with respect to the global heat and momentum transport, temperature and velocity profiles, as well as the dynamics of the large-scale circulation (LSC). The DNS reveal that the twisting and sloshing of the LSC at small inclination angles periodically affects the instantaneous heat transport (up to $\pm 44 \%$ of the mean heat transport). The twisted LSC is associated with a weak heat transport, while the sloshing mode that brings together the hot and cold streams of the LSC is associated with a strong heat transport. The experiments show that the heat transport scales as $N u \sim R a^{0.22}$ in both limiting cases (RBC and VC) for Rayleigh numbers around $R a \approx 10^{7}$, while any inclination of the cell, $0<\beta \leqslant 90^{\circ}$, leads to an increase of $\mathrm{Nu}$.

Key words: Bénard convection, convection in cavities, turbulent convection

\section{Introduction}

Elucidation of the mechanisms of turbulent thermal convection in very-low-Prandtlnumber fluids is crucial for our understanding of the universe and the advancement

$†$ Email address for correspondence: lukas.zwirner@ds.mpg.de 
of cooling technology. Turbulent thermal convection takes place, for example, on the surfaces of stars, where the Prandtl number $(P r)$ varies from $10^{-8}$ to $10^{-4}$ (Spiegel 1962; Hanasoge, Gizon \& Sreenivasan 2016). Furthermore, turbulent thermal convection in liquid metals $(\operatorname{Pr} \ll 1)$ is relevant in engineering applications, especially in cooling systems of tokamaks and fast breeder reactors (Zhilin et al. 2009; Belyaev et al. 2013). Liquid sodium is of particular interest because of its very low Prandtl number $(\operatorname{Pr} \approx 0.009)$ and it is widely used as cooling agent in fast neutron reactors (Heinzel et al. 2017).

One classical model of thermal convection is Rayleigh-Bénard convection (RBC), where the fluid is confined between a heated lower plate and a cooled upper plate, and the main driving force is buoyancy. The temperature inhomogeneity varies the fluid density, which in presence of gravity leads to the convective fluid motion. For reviews on RBC we refer to Bodenschatz, Pesch \& Ahlers (2000), Ahlers, Grossmann \& Lohse (2009), Lohse \& Xia (2010) and Chillà \& Schumacher (2012).

Thermal convection inevitably arises in the case of a horizontal temperature gradient. This is known as vertical convection (VC), convection in cavities or side-heated convection. In vertical convection, the heated and cooled plates are located parallel to the gravity vector and shear plays the key role, see $\mathrm{Ng}$ et al. $(2015,2017)$ and Shishkina (2016).

The concept of inclined convection (IC) is a generalisation of $\mathrm{RBC}$ and VC, i.e. the fluid layer between the parallel plates is tilted with respect to the direction of gravity, and both buoyancy and shear act on the flow. This type of convection was studied previously by Daniels, Wiener \& Bodenschatz (2003), Chillà et al. (2004), Sun, Xi \& Xia (2005), Ahlers, Brown \& Nikolaenko (2006b), Riedinger et al. (2013), Weiss \& Ahlers (2013) and Langebach \& Haberstroh (2014), and more recently by Frick et al. (2015), Mamykin et al. (2015), Vasil'ev et al. (2015), Kolesnichenko et al. (2015), Shishkina \& Horn (2016), Teimurazov \& Frick (2017), Mandrykin \& Teimurazov (2019), Khalilov et al. (2018) and Zwirner \& Shishkina (2018).

In thermal convection, the global flow structures and heat and momentum transport are determined mainly by the following system parameters: the Rayleigh number $R a$, the Prandtl number $\mathrm{Pr}$ and the aspect ratio of the container $\Gamma$. These are defined as

$$
R a \equiv \alpha g \Delta L^{3} /(\kappa \nu), \quad P r \equiv v / \kappa, \quad \Gamma \equiv D / L, \quad(1.1 a-c)
$$

respectively. Here, $\alpha$ denotes the isobaric thermal expansion coefficient, $v$ the kinematic viscosity, $\kappa$ the thermal diffusivity of the fluid, $g$ the acceleration due to gravity, $\Delta \equiv T_{+}-T_{-}$the difference between the temperatures at the heated plate $\left(T_{+}\right)$and at the cooled plate $\left(T_{-}\right), L$ the distance between the plates and $D$ the diameter of the plates.

The main response characteristics of a natural convective system are the mean total heat flux across the heated/cooled plates, $q$, normalised by the conductive part of the total heat flux, $\hat{q}$, i.e. the Nusselt number $N u$, and the Reynolds number $R e$ :

$$
N u \equiv q / \hat{q}, \quad \operatorname{Re} \equiv L U / \nu .
$$

Here, $U$ is the reference velocity, which is usually determined by either the maximum of the time-averaged velocity along the plates or by $\langle\boldsymbol{u} \cdot \boldsymbol{u}\rangle^{1 / 2}$, i.e. it is based on the mean kinetic energy; $\boldsymbol{u}$ is the velocity vector field and $\langle\cdot\rangle$ denotes the average in time and over the whole convection cell. Note that, even for a fixed set-up in natural thermal convection with no additional shear imposed on the system, the scaling relations of the mean heat and momentum transport, represented by $N u$ and 
$R e$, with the input parameters $R a$ and $P r$, are not universal and are influenced by non-Oberbeck-Boussinesq (NOB) effects, see Kraichnan (1962), Grossmann \& Lohse (2000), Ahlers et al. (2006a, 2009), Lohse \& Xia (2010), Shishkina, Grossmann \& Lohse (2016a), Shishkina, Weiss \& Bodenschatz (2016b) and Weiss et al. (2018).

Here, one should note that, apart from $\operatorname{Pr}$ and $R a$, the geometrical confinement of the convection cell also determines the strength of the heat transport (Huang et al. 2013; Chong et al. 2015; Chong \& Xia 2016). Thus, in experiments by Huang et al. (2013) for $P r=4.38$, an increase of $N u$ due to the cell confinement was obtained, while in the direct numerical simulations (DNS) by Wagner \& Shishkina (2013) for $\operatorname{Pr}=0.786$, the heat and mass transport gradually reduced with increasing confinement. This virtual contradiction was recently resolved in Chong et al. (2018). It was found that $\operatorname{Pr}$ determines whether the optimal $\Gamma$, at which the maximal heat transport takes place, exists or not. For $\operatorname{Pr}>0.5\left(R a=10^{8}\right)$ an enhancement of $\mathrm{Nu}$ was observed, where the optimal $\Gamma$ decreases with increasing $\operatorname{Pr}$, but for $\operatorname{Pr} \leqslant 0.5$ a gradual reduction of the heat transport with increasing confinement was obtained. For all $P r$, the confinement induced friction causes a reduction of $R e$.

In the general case of inclined thermal convection, the cell inclination angle $\beta$ ( $\beta=0^{\circ}$ in $\mathrm{RBC}$ and $\beta=90^{\circ}$ in $\mathrm{VC}$ ) is an influential input parameter of the convective system, apart from $\mathrm{Ra}, \mathrm{Pr}$ and the geometry of the container. Experimental studies of inclined thermal liquid-sodium convection in cylinders of different aspect ratios, showed that the convective heat transfer between the heated and cooled surfaces of the container is most efficient neither in a standing position of the cylinder $\left(\beta=0^{\circ}\right)$, nor in a lying position $\left(\beta=90^{\circ}\right)$, but at an inclined position for a certain intermediate value of $\beta, 0^{\circ}<\beta<90^{\circ}$, see Vasil'ev et al. (2015) for $L=20 D$, Mamykin et al. (2015) for $L=5 D$ and Khalilov et al. (2018) for $L=D$. Moreover, these experiments showed that, for $\operatorname{Pr} \ll 1$ and $R a \gtrsim 10^{9}$, any tilt $\beta, 0^{\circ}<\beta \leqslant 90^{\circ}$, of the cell leads to a larger mean heat flux $(\mathrm{Nu})$ than in RBC, at similar values of $R a$ and $P r$. Note that the effect of the cell tilting on the convective heat transport in low- $P r$ fluids is very different from that in the case of large $\operatorname{Pr}$ (Shishkina \& Horn 2016). For example, for $\operatorname{Pr} \approx 6.7$ and $R a \approx 4.4 \times 10^{9}$, a monotonic reduction of $N u$ with increasing $\beta$ in the interval $\beta \in\left[0^{\circ}, 90^{\circ}\right]$ takes place, as it was obtained in measurements by Guo et al. (2015).

One should mention that there are only a few experimental and numerical studies of IC in a broad range of $\beta$, whereas most of the investigations of the cell-tilt effects on the mean heat transport were conducted in a narrow region of $\beta$ close to $0^{\circ}$ and mainly for large- $P r$ fluids. These studies showed generally a small effect of $\beta$ on $N u$, reflected in a tiny reduction of $N u$ with increasing $\beta$ close to $\beta=0^{\circ}$, see Ciliberto, Cioni \& Laroche (1996), Cioni, Ciliberto \& Sommeria (1997), Chillà et al. (2004), Sun et al. (2005), Ahlers et al. (2006b), Roche et al. (2010) and Wei \& Xia (2013). A tiny local increase of $N u$ with a small inclination of the RBC cell filled with a fluid of $\operatorname{Pr}>1$ is possible only when a two-roll form of the global large-scale circulation (LSC) is present in RBC, which usually almost immediately transforms into a singleroll form of the LSC with any inclination (Weiss \& Ahlers 2013). The single-roll LSC is known to be more efficient in the heat transport than its double-roll form, as was proved in the measurements (Xi \& Xia 2008; Weiss \& Ahlers 2013) and DNS (Zwirner \& Shishkina 2018).

The IC in a broad range of the inclination angle $\beta$ (from $0^{\circ}$ to $90^{\circ}$ ) in liquid sodium has been studied so far by Frick et al. (2015), Mamykin et al. (2015), Vasil'ev et al. (2015), Kolesnichenko et al. (2015) and Khalilov et al. (2018). These sodium experiments were conducted in relatively long cylinders, in which the scaling 
exponents are essentially increased due to the geometrical confinement. For RBC in a cylinder with $L=5 D$, Frick et al. (2015) reported $N u \sim R a^{0.4}$ and for RBC in a very long cylinder with $L=20 D$, Mamykin et al. (2015) obtained $N u \sim R a^{0.77}$. In both studies, also IC in liquid sodium for $\beta=45^{\circ}$ was investigated, and the following scaling laws were obtained for this inclination angle: $N u \sim R a^{0.54}$ for $L=5 D$ and $N u \sim R a^{0.7}$ for $L=20 D$. Note that in both IC measurements, much higher mean heat fluxes were obtained, compared to those in VC or RBC configurations.

Thus, all available experimental and numerical results on IC show that the $N u(\beta) / N u(0)$ dependence is a complex function of $\operatorname{Ra}, \operatorname{Pr}$ and $\Gamma$, which cannot be represented as a simple combination of their power functions.

An analogy can be seen between the IC flows and convective flows, which occur from the imposed temperature differences at both the horizontal and vertical surfaces of a cubical container. With a different balance between the imposed horizontal and vertical temperature gradients, where the resulting effective temperature gradient has non-vanishing horizontal and vertical components, one can mimic the IC flows at different inclination angles. Experimental studies on these type of convective flows were conducted by Zimin, Frik \& Shaidurov (1982).

Although there is no scaling theory for general IC, for the limiting configurations of IC $\left(\beta=0^{\circ}\right.$ and $\left.\beta=90^{\circ}\right)$ and sufficiently wide heated/cooled plates, there are theoretical studies of the scaling relations of $N u$ and $R e$ with $\operatorname{Pr}$ and $R a$. For RBC $(\beta=0)$, Grossmann \& Lohse (2000, 2001, 2011) (hereafter GL) developed a scaling theory which is based on a decomposition into boundary-layer (BL) and bulk contributions of the time- and volume-averaged kinetic $\left(\epsilon_{u}\right)$ and thermal $\left(\epsilon_{\theta}\right)$ dissipation rates, for which analytical relations with $N u, R a$ and $\operatorname{Pr}$ exist. Equating $\epsilon_{u}$ and $\epsilon_{\theta}$ to their estimated either bulk or BL contributions and employing in the BL dominated regimes the Prandtl-Blasius BL theory (Prandtl 1905; Blasius 1908; Landau \& Lifshitz 1987; Schlichting \& Gersten 2000), theoretically possible limiting scaling regimes were derived. The theory allows us to predict $N u$ and $R e$ in RBC if the pre-factors fitted with the latest experimental and numerical data are used, see Stevens et al. (2013) and Shishkina et al. (2017).

In the other limiting case of IC, which is $\operatorname{VC}\left(\beta=90^{\circ}\right)$, the mean kinetic dissipation rate $\epsilon_{u}$ cannot be derived analytically from $\operatorname{Ra}, \operatorname{Pr}$ and $N u$, and this impedes an extension of the GL theory to VC. However, for the case of laminar free convection between two differentially heated plates (i.e. VC), it is possible to derive the dependences of $R e$ and $N u$ on $R a$ and $P r$ from the BL equations, under the assumption that a similarity solution exists (Shishkina 2016). Although this problem is solved for the laminar case, to our knowledge, there is no theoretical model to predict $N u$ and $R e$ in turbulent VC. It is expected, however, that in the asymptotic regime of high $R a$, the scaling exponent in the $N u$ versus $R a$ and $R e$ versus $R a$ scalings is $1 / 2$, as in $\mathrm{RBC}(\mathrm{Ng}$ et al. 2018).

Generally, the dependences of $N u$ and $R e$ on $R a$ and $P r$ in VC have been less investigated than those in RBC. For similar cell geometry and ranges of $R a$ and $P r$, not only the heat transport in VC differs quantitatively from that in RBC (Bailon-Cuba et al. 2012; Wagner \& Shishkina 2013, 2015; Ng et al. 2015), but the VC and RBC flows can even be in different states. For example, for $\operatorname{Pr}=1, \operatorname{Ra}=10^{8}$ and a cylindrical container of $\Gamma=1$, the VC flow is steady, while the RBC flow is already turbulent (Shishkina \& Horn 2016). Previous experimental and numerical studies of free thermal convection under an imposed horizontal temperature gradient (i.e. VC) reported the scaling exponent $\gamma$ in the power law $N u \sim R a^{\gamma}$, varying from $1 / 4$ to $1 / 3$. In laminar VC, it is approximately $1 / 4$ (Schmidt \& Beckmann 1930; Lorenz 
1934; Saunders 1939; Churchill \& Chu 1975), being slightly larger for very small $R a$, where the geometrical cell confinement influences the heat transport (Versteegh \& Nieuwstadt 1999; Yu, Li \& Ecke 2007; Kis \& Herwig 2012; Ng et al. 2015). The scaling exponent $\gamma$ is also larger for very high $R a$, where, with growing $R a$, the $\mathrm{VC}$ flows become first transitional ( $\mathrm{Ng}$ et al. 2017) and later on fully turbulent (Fujii et al. 1970; George \& Capp 1979). Note that all the mentioned experiments and simulations of $\mathrm{VC}$ were conducted for fluids of $\mathrm{Pr}$ about or larger than 1.

For the case of small $P r$, in the experiments by Frick et al. (2015) and Mamykin et al. (2015) on turbulent $\mathrm{VC}$ in liquid sodium $(P r \approx 0.01)$ in elongated cylinders, significantly larger scaling exponents were observed, due to the geometrical confinement. Thus, for a cylinder with $L=5 D$ and the Rayleigh numbers, based on the cylinder diameter, up to $10^{7}$, Frick et al. (2015) obtained $N u \sim R a^{0.43}$ and $R e \sim G r^{0.44}$, where $G r$ is the Grashof number, $G r \equiv R a / P r$. For an extremely strong geometrical confinement, namely, for a cylindrical convection cell with $L=20 D$, and a similar Rayleigh number range, Mamykin et al. (2015) found $N u \sim R a^{0.95}$ and $\operatorname{Re} \sim G r^{0.63}$.

There exist a few measurements of the scaling relations of $N u$ versus $R a$ in liquidmetal Rayleigh-Bénard convection (without any cell inclination). For mercury ( $\mathrm{Pr} \approx$ 0.024 ), it was reported $N u \sim R a^{0.27}$ for $2 \times 10^{6}<R a<8 \times 10^{7}$ by Takeshita et al. (1996), $N u \sim R a^{0.26}$ for $7 \times 10^{6}<R a<4.5 \times 10^{8}$ and $N u \sim R a^{0.20}$ for $4.5 \times 10^{8}<R a<$ $2.1 \times 10^{9}$ by Cioni et al. (1997) and $N u \sim R a^{0.29}$ for $2 \times 10^{5}<R a<7 \times 10^{10}$ by Glazier et al. (1999). For liquid gallium $(\operatorname{Pr} \approx 0.025)$, King \& Aurnou (2013) measured $N u \sim$ $R a^{0.25}$ for $2 \times 10^{6}<R a<10^{8}$, and for GaInSn $(P r=0.029)$, Zürner et al. (2019) measured $N u \sim R a^{0.27}$ for $4 \times 10^{6}<R a<6 \times 10^{7}$. For liquid sodium $(\operatorname{Pr}=0.006)$, Horanyi, Krebs \& Müller (1999) measured $N u \sim R a^{0.25}$ for $2 \times 10^{4}<R a<5 \times 10^{6}$.

Conducting accurate DNS of natural thermal convection at high $R a$ and very low $P r$ is very challenging, since it requires very fine meshes in space and short steps in time due to the necessity to resolve the Kolmogorov microscales in the bulk of the flows as well in the viscous BLs (Shishkina et al. 2010). When $\operatorname{Pr} \ll 1$, the thermal diffusion, represented by $\kappa$, is much larger than the momentum diffusion, represented by $v$, and therefore, the viscous BLs become extremely thin at large $R a$. Thus, there exist only a few DNS of thermal convection for a combination of large $R a$ and very low Prandtl numbers, $P r \leqslant 0.025$, which, moreover, have been conducted exclusively for the Rayleigh-Bénard configuration (Scheel \& Schumacher 2016, 2017; Schumacher et al. 2016; Horn \& Schmid 2017; Vogt et al. 2018).

DNS of turbulent flows at extremely large $G r$ and extremely small Prandtl numbers, for example convective flow of magnesium that develops in a titanium reduction reactor (characterised by $\mathrm{Gr}$ of the order of $10^{12}$ ), is currently unrealisable. Therefore, further development of reduced mathematical models is still required. Computational codes for large-eddy simulations (LES) of turbulent thermal convection in liquid metals, verified against the corresponding experiments and DNS, can be useful in solving this kind of problem (Teimurazov, Frick \& Stefani 2017).

Although knowledge on thermal convection in liquid metals is required for the development of safe and efficient liquid metal heat exchangers, the experimental database of the corresponding measurements remains quite restricted due to the known difficulties in conducting thermal measurements in liquid metals. Apart from general problems, occurring from the high temperature and aggressivity of liquid sodium, natural convective flows are known to be relatively slow and are very sensitive to the imposed disturbances. While probe measurements in the core part of pipe and channel flows are possible (Heinzel et al. 2017; Onea et al. 2017a,b), since 


$\begin{array}{lcc} & \begin{array}{c}\lambda \\ \left(\mathrm{W} \mathrm{mK} \mathrm{mK}^{-1}\right)\end{array} & \begin{array}{c}\kappa \times 10^{6} \\ \left(\mathrm{~m}^{2} \mathrm{~s}^{-1}\right)\end{array} \\ \text { Stainless steel } & 17 & 4.53 \\ \mathrm{Na} & 84.6 & 66.5 \\ \mathrm{Cu} & 391.5 & 111\end{array}$

TABLE 1 . The thermal conductivity $\lambda$ and the thermal diffusivity $\kappa$ of stainless steel, liquid sodium $(\mathrm{Na})$ and copper $(\mathrm{Cu})$ at the mean temperature of the experiment of approximately $410 \mathrm{~K}$.

the flow is influenced only downstream, they will unavoidably induce too strong disturbances in the case of natural thermal convection.

Thus, IC measurements in liquid sodium at large $R a$ and the corresponding precise numerical simulations are in great demand and, therefore, we devote our present work to this topic. Combining experiments, DNS and LES, we aim to paint a complementary picture of turbulent thermal liquid-sodium convection. Using these three viewpoints on liquid-sodium convection helps us to overcome their individual difficulties and simultaneously verify their observations.

The outline of this work is as follows: in $\S 2$ we introduce the experiment, DNS, LES and methods of data analysis. Section 3 presents the obtained results on how the global flow structures (mainly LSC) and their evolution in time affect the heat and momentum transport in case of liquid-sodium convection. An analogy of the global flow structures and global heat transport in flows with similar values of $\mathrm{RaPr}$ is also discussed there. The final section summarises our results.

\section{Methods}

\subsection{Experiment}

All experimental data presented in this paper are obtained at the experimental facility described in detail in Khalilov et al. (2018). The convection cell is made of a stainless steel pipe with a $3.5 \mathrm{~mm}$ thick wall. The inner length of the convection cell is $L=216 \mathrm{~mm}$ and the inner diameter $D=212 \mathrm{~mm}$. Both end faces of the convection cell are separated from the heat exchanger chambers by $1 \mathrm{~mm}$ thick copper discs, see a sketch in figure 1. The convection cell is filled with liquid sodium. The heat exchanger chambers are filled also with liquid sodium and the temperature there is kept constant. The thin end-face copper plates are intensively washed with liquid sodium from the chamber sides, ensuring homogeneous temperature distributions at their surfaces (Kolesnichenko et al. 2017). The entire set-up is placed on a swing frame, so that the convection cell can be tilted from a vertical position to a horizontal one. Inclination of the convection cell is then characterised by the angle $\beta$ between the vertical and the cylinder axis, see figure 1.

Obviously, the boundary conditions in a real liquid-metal experiment and the idealised boundary conditions that are considered in numerical simulations, are different. For example, in the simulations, the cylindrical sidewall is assumed to be adiabatic, while in the real experiment it is made from $3.5 \mathrm{~mm}$ thick stainless steel and is additionally covered by a $30 \mathrm{~mm}$ thick layer of mineral wool. In table 1 , the thermal characteristics of stainless steel, liquid sodium and copper are presented. One can see that the thermal diffusivity of the stainless steel, although being smaller compared to that of liquid sodium, is not negligible. Copper, which is known to be 


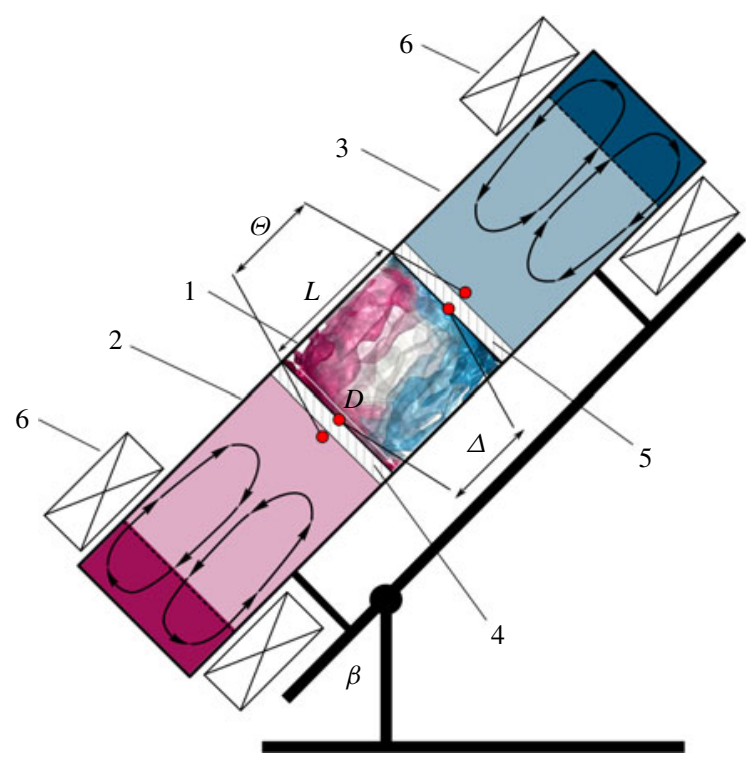

FIGURE 1. Sketch of the experimental facility, which consists of: (1) a cylindrical convection cell, (2) a hot heat exchanger chamber, (3) a cold heat exchanger chamber, (4) a heated copper plate, (5) a cooled copper plate and (6) inductor coils. The convection cell (1) and heat exchangers $(2,3)$ are filled with liquid sodium. $D$ is the diameter and $L$ the length of the cylindrical convection cell $(1), \beta$ is the cell inclination angle, $\Theta$ the temperature drop between the hot and cold heat exchanger chambers, $\Delta$ the resulting temperature drop between the inner surfaces of the heated and cooled plates of the convection cell (1).

the best material for the heat exchangers in the experiments with moderate or high $\operatorname{Pr}$ fluids, has the thermal diffusivity of the same order as the thermal diffusivity of sodium. Therefore, massive copper plates would not provide a uniform temperature at the surfaces of the plates (Kolesnichenko et al. 2015). To avoid this undesirable inhomogeneity, in our experiment, instead of thick copper plates, we use rather thin ones, which are intensively washed from the outside by liquid sodium of prescribed temperature (see figure 1). The latter process takes place in two heat exchanger chambers, a hot one and a cold one, which are equipped with induction coils. A sodium flow in each heat exchanger chamber is provided by a travelling magnetic field. The induction coils are attached near the outer end faces of the corresponding heat exchanger, thus ensuring that the electromagnetic influence of the inductors on the liquid metal in the convection cell is negligible (Kolesnichenko et al. 2017). Typical velocities of the sodium flows in the heat exchangers are approximately $1 \mathrm{~m} \mathrm{~s}^{-1}$, being an order of magnitude higher than the convective velocity inside the convection cell.

In all conducted experiments, the mean temperature of liquid sodium inside the convection cell is approximately $T_{m}=139.8^{\circ} \mathrm{C}$, for which the Prandtl number equals $\operatorname{Pr} \approx 0.0093$. Each experiment is performed for a prescribed and known applied temperature difference $\Theta=T_{\text {hot }}-T_{\text {cold }}$, where $T_{\text {hot }}$ and $T_{\text {cold }}$ are the time-averaged temperatures of sodium in, respectively, the hot and cold heat exchanger chambers, which are measured close to the copper plates (see figure 1). 
In any hot liquid-metal experiment, there exist unavoidable heat losses due to the high temperature of the set-up. To estimate the corresponding power losses $Q_{\text {loss }}$, one needs to measure additionally the power, which is required to maintain the same mean temperature $T_{m}$ of liquid sodium inside the convection cell, but under the condition of equal temperatures in both heat exchanger chambers, that is, for $\Theta=0$. From $Q_{\text {loss }}$ and the total power consumption $Q$ in the convective experiment at $\Theta \neq 0$, one calculates the effective power $Q_{\text {eff }}=Q-Q_{\text {loss }}$ in any particular experiment.

Although the thermal resistance of the two thin copper plates themselves is negligible, the sodium-copper interfaces provide additional effective thermal resistance of the plates mainly due to inevitable oxide films. The temperature drop $\Delta_{p l}$ through both copper plates covered by the oxide films could be calculated then from $\Delta_{p l}=Q_{e f f} R_{p l}$, as soon as the effective thermal resistance of the plates, $R_{p l}$, is known. Note that for a fixed mean temperature $T_{m}$, the value of $R_{p l}$ depends on $\Theta$, since the effective thermal conductivities of the two plates are different due to their different temperatures.

The values of $R_{p l}(\Theta)$ for different $\Theta$ are calculated in a series of auxiliary measurements for the case of $\beta=0$ and stable temperature stratification, where the heat is applied from above, to suppress convection. In this purely conductive case, the effective thermal resistance of the plates, $R_{p l}$, can be calculated from $\Theta$ and the measured effective power $\bar{Q}_{\text {eff }}$ from the relation $\Theta=\bar{Q}_{\text {eff }}\left(R_{N a}+R_{p l}\right)$, where the thermal resistance of the liquid sodium equals $R_{N a}=L /\left(\lambda_{N a} S\right)$ with $\lambda_{N a}$ being the liquid-sodium thermal conductivity and $S=\pi R^{2}$ with the cylinder radius $R$.

Using the above measured effective thermal resistances of the plates, $R_{p l}(\Theta)$, in any convection experiment for a given $R a$ and $\beta$ and the mean temperature $T_{m}$, the previously unknown temperature drop $\Delta$ inside the convection cell can be calculated from $\Theta, R_{p l}(\Theta)$ and measured $Q_{e f f}$ as follows:

$$
\Delta=\Theta-\Delta_{p l}, \quad \Delta_{p l}=Q_{e f f} R_{p l}
$$

Note that in all our measurements in liquid sodium, for all considered inclination angles of the convection cell, the obtained mean temperature and the temperature drop within the cell equal, respectively, $T_{m} \approx 139.8^{\circ}$ and $\Delta \approx 25.3 \mathrm{~K}$.

The Nusselt number is then calculated as

$$
N u=\frac{L Q_{e f f}}{\lambda_{N a} S \Delta} .
$$

For a comparison of the experimental results with the DNS and LES results, where the temperatures at the plates inside the convection cell are known a priori, the experimental temperature at the hot plate, $T_{+}$, and that at the cooled plate, $T_{-}$, are calculated as follows:

$$
T_{+}=T_{m}+\Delta / 2, \quad T_{-}=T_{m}-\Delta / 2 .
$$

The Rayleigh number in the experiment is evaluated as

$$
R a \equiv \alpha g \Delta D^{4} /(L \kappa v),
$$

which slightly differs from the value defined in (1.1), since $D$ is slightly smaller than $L$. Thus, for $\alpha=2.56 \times 10^{-4} \mathrm{~K}^{-1}, g=9.81 \mathrm{~m} \mathrm{~s}^{-2}, \Delta=25.3 \mathrm{~K}, v=6.174 \times$ $10^{-7} \mathrm{~m}^{2} \mathrm{~s}^{-1}$ and $\kappa=6.651 \times 10^{-5} \mathrm{~m}^{2} \mathrm{~s}^{-1}$, the Rayleigh number equals $R a=(1.42 \pm$ $0.03) \times 10^{7}$. 
(a)

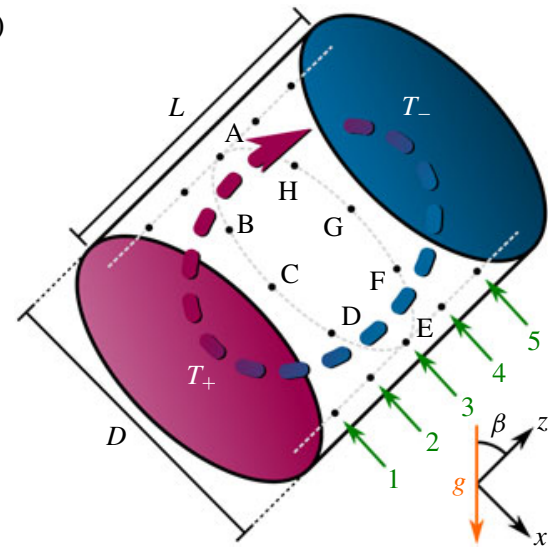

(b)

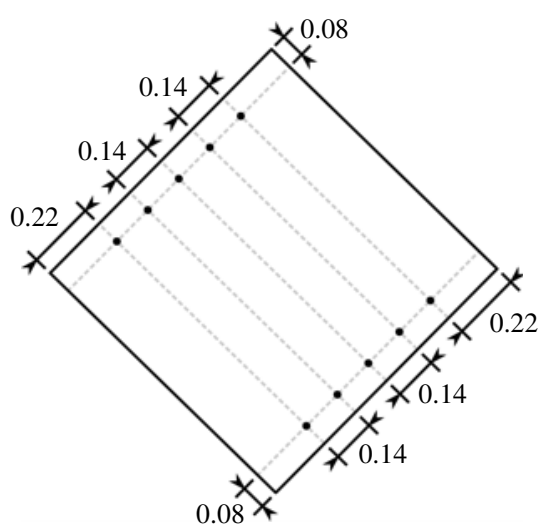

FIGURE 2. (a) Sketch of an inclined convection cell. $D$ is the diameter and $L$ the height of the cylindrical sample, $\beta$ is the inclination angle, $T_{+}\left(T_{-}\right)$the temperature of the heated (cooled) surfaces. Positioning and naming of the 40 probes inside the cylinder, as considered in the DNS (all combinations of the azimuthal locations A, B, C, D, E, F, G, $\mathrm{H}$ and circles $1, \ldots, 5$ ) and 28 probes in the experiments (all combinations of the eight locations $\mathrm{A}, \ldots, \mathrm{H}$ for the circles 1,3 and 5 plus four additional probes: A2, A4, E2, E4). Note that the azimuthal locations are shown only for the circle 3, not to overload the sketch. For any inclination angle $\beta>0^{\circ}$, the upper azimuthal location is A. (b) Sketch of a central vertical cross-section of the set-up from figure $(a)$, with shown distances (normalised with $L=D$ ) between the neighbouring probes and between the probes and the sidewall of the cylindrical convection cell.

For a deep analysis of the convective liquid-sodium flows, the convection cell is equipped with 28 thermocouples, each with an isolated junction of $1 \mathrm{~mm}$. The thermocouples are located on 8 lines aligned parallel to the cylinder axis (see figure 2). The azimuthal locations of these lines are distributed with an equal azimuthal step of $45^{\circ}$ and are marked in figure 2 by capital letters $\mathrm{A}$ to $\mathrm{H}$ (counterclockwise, if looking from the cold end face). The line $\mathrm{A}$ has the upper position if $\beta>0^{\circ}$. On each of the eight lines (A to $\mathrm{H}$ ), 3 or 5 thermocouples are placed. Thus, all thermocouples are located in five cross-sections of the convection cell, which are parallel to the end faces. The thermocouples are installed inside the convection cell at the same distance of $17 \mathrm{~mm}$ from the inner cylinder sidewall and thus are located on five circles, which are marked in figure 2 with numbers $1, \ldots, 5$. The circles 1,3 and 5 include eight thermocouples, and the circles 2 and 4 only two thermocouples (A and E).

In this paper, besides the measurements by Khalilov et al. (2018), where a single Rayleigh number $R a=(1.42 \pm 0.03) \times 10^{7}$ was considered, we present and analyse also new experimental data, which are obtained for a certain range of the Rayleigh number, based on different imposed temperature gradients.

\subsection{Direct numerical simulations}

The problem of inclined thermal convection within the Oberbeck-Boussinesq (OB) approximation, which is studied in the DNS, is defined by the following Navier-Stokes, temperature and continuity equations in cylindrical coordinates $(r, \phi, z)$ :

$$
D_{t} \boldsymbol{u}=v \nabla^{2} \boldsymbol{u}-\nabla p+\alpha g\left(T-T_{0}\right) \hat{\boldsymbol{e}}
$$




$$
\begin{gathered}
D_{t} T=\kappa \nabla^{2} T, \\
\nabla \cdot \boldsymbol{u}=0,
\end{gathered}
$$

where $D_{t}$ denotes the substantial derivative, $\boldsymbol{u}=\left(u_{r}, u_{\phi}, u_{z}\right)$ the velocity vector field, with the component $u_{z}$ in the direction $z$, which is orthogonal to the plates, $p$ is the reduced kinetic pressure, $T$ the temperature, $T_{0}=\left(T_{+}+T_{-}\right) / 2$ and $\hat{\boldsymbol{e}}$ is the unit vector, $\hat{\boldsymbol{e}}=(-\sin (\beta) \cos (\phi), \sin (\beta) \sin (\phi), \cos (\beta))$. Within the considered OB approximation, it is assumed that the fluid properties are independent of the temperature and pressure, apart from the buoyancy term in the Navier-Stokes equation, where the density is taken linearly dependent on the temperature.

These equations are non-dimensionalised by using the cylinder radius $R$, the free-fall velocity $U_{f}$, the free-fall time $t_{f}$,

$$
U_{f} \equiv(\alpha g R \Delta)^{1 / 2}, \quad t_{f}=R(\alpha g R \Delta)^{-1 / 2}
$$

and the temperature drop between the heated plate and the cooled plate, $\Delta$, as units of length, velocity, time and temperature, respectively.

To close the system (2.5)-(2.7), the following boundary conditions are considered: no slip for the velocity at all boundaries, $\boldsymbol{u}=0$, constant temperatures $\left(T_{-}\right.$or $\left.T_{+}\right)$ at the face ends of the cylinder and adiabatic boundary condition at the sidewall, $\partial T / \partial r=0$.

The resulting dimensionless equations are solved numerically with the finite-volume computational code GOLDFISH, which uses high-order interpolation schemes in space and a direct solver for the pressure (Kooij et al. 2018). No turbulence model is applied in the simulations. The utilised staggered computational grids of approximately $1.5 \times$ $10^{8}$ nodes, which are clustered near all rigid walls, are sufficiently fine to resolve the Kolmogorov microscales (see table 2). Statistical averaging is usually carried out for several hundred free-fall time units, which is sufficient not only for integral quantities like the Nusselt number and Reynolds number to converge, but also for the flow fields. The exceptions are the most expensive DNS for liquid sodium. Statistical averaging in our liquid-sodium DNS is, however, the longest known for such low Prandtl numbers and the integral quantities and also the velocity and temperature profiles are converged to a reasonable degree.

Since simulations on such fine meshes are extremely expensive, only four inclination angles are considered for the main case of $\operatorname{Pr}=0.0094$ and $\operatorname{Ra}=1.67 \times 10^{7}$, which are $\beta=0^{\circ}, \beta=36^{\circ}, \beta=72^{\circ}$ and $\beta=90^{\circ}$. To study similarities of the flows with respect to the global heat transport and global flow structures for an almost constant Grashof number, $G r \equiv R a / P r$, and for a fixed value of $R a P r$, some additional DNS of IC were conducted for the combinations of $P r=0.094$ with $R a=1.67 \times 10^{6}$ and $\operatorname{Pr}=1$ with $R a=10^{9}$. Note that the Grashof number of the auxiliary DNS $\left(G r=10^{9}\right)$ is slightly different from that of the liquid-sodium DNS $\left(G r=1.78 \times 10^{9}\right)$. In the former additional DNS, eight different inclination angles are considered, while in the latter additional DNS, 11 different values of $\beta$ are examined (see table 2). The computational grids, used in the auxiliary DNS for similar $\operatorname{Ra} P r$, contain only three nodes within each viscous boundary layer, however, a convergence study, using twice as many nodes in each direction, for three different inclination angles demonstrated a deviation in $\mathrm{Nu}$ and $\mathrm{Ra}$ within $1 \%$. 


\begin{tabular}{|c|c|c|c|c|c|c|c|c|c|c|c|}
\hline & $R a$ & $P r$ & $\beta$ (deg.) & $t_{\text {avg }} / t_{f}$ & $N_{r}$ & $N_{\phi}$ & $N_{z}$ & $\mathcal{N}_{t h}$ & $\mathcal{N}_{v}$ & $\delta_{u} / L$ & $\delta_{t h} / L$ \\
\hline \multirow[t]{4}{*}{ DNS } & $1.67 \times 10^{7}$ & 0.0094 & 0 & 157.2 & 384 & 512 & 768 & 82 & 7 & $3.4 \times 10^{-3}$ & $5.2 \times 10^{-2}$ \\
\hline & & & 36 & 89.0 & 384 & 512 & 768 & 67 & 7 & $3.2 \times 10^{-3}$ & $4.1 \times 10^{-2}$ \\
\hline & & & 72 & 72.0 & 384 & 512 & 768 & 69 & 7 & $3.2 \times 10^{-3}$ & $4.2 \times 10^{-2}$ \\
\hline & & & 90 & 66.8 & 384 & 512 & 768 & 77 & 7 & $3.4 \times 10^{-3}$ & $4.8 \times 10^{-2}$ \\
\hline \multirow[t]{10}{*}{ LES } & $1.5 \times 10^{7}$ & 0.0093 & 0 & 460.5 & 100 & 160 & 200 & 32 & 3 & $3.3 \times 10^{-3}$ & $5.4 \times 10^{-2}$ \\
\hline & & & 10 & 629.4 & 100 & 160 & 200 & 31 & 3 & $3.2 \times 10^{-3}$ & $5.2 \times 10^{-2}$ \\
\hline & & & 20 & 806.0 & 100 & 160 & 200 & 29 & 3 & $3.1 \times 10^{-3}$ & $4.9 \times 10^{-2}$ \\
\hline & & & 30 & 468.2 & 100 & 160 & 200 & 27 & 3 & $3.1 \times 10^{-3}$ & $4.4 \times 10^{-2}$ \\
\hline & & & 40 & 468.2 & 100 & 160 & 200 & 27 & 3 & $3.1 \times 10^{-3}$ & $4.3 \times 10^{-2}$ \\
\hline & & & 50 & 928.8 & 100 & 160 & 200 & 26 & 3 & $3.1 \times 10^{-3}$ & $4.2 \times 10^{-2}$ \\
\hline & & & 60 & 560.3 & 100 & 160 & 200 & 27 & 3 & $3.1 \times 10^{-3}$ & $4.2 \times 10^{-2}$ \\
\hline & & & 70 & 460.5 & 100 & 160 & 200 & 27 & 3 & $3.1 \times 10^{-3}$ & $4.3 \times 10^{-2}$ \\
\hline & & & 80 & 537.3 & 100 & 160 & 200 & 28 & 3 & $3.2 \times 10^{-3}$ & $4.6 \times 10^{-2}$ \\
\hline & & & 90 & 652.4 & 100 & 160 & 200 & 30 & 3 & $3.3 \times 10^{-3}$ & $5.0 \times 10^{-2}$ \\
\hline \multirow[t]{8}{*}{ DNS } & $1.67 \times 10^{6}$ & 0.0940 & 0 & 4000 & 95 & 128 & 192 & 13 & 3 & $1.21 \times 10^{-2}$ & $6.1 \times 10^{-2}$ \\
\hline & & & 9 & 1000 & 95 & 128 & 192 & 12 & 3 & $1.24 \times 10^{-2}$ & $5.7 \times 10^{-2}$ \\
\hline & & & 18 & 2000 & 95 & 128 & 192 & 12 & 3 & $1.19 \times 10^{-2}$ & $5.4 \times 10^{-2}$ \\
\hline & & & 27 & 1000 & 95 & 128 & 192 & 11 & 3 & $1.15 \times 10^{-2}$ & $5.2 \times 10^{-2}$ \\
\hline & & & 36 & 2000 & 95 & 128 & 192 & 11 & 3 & $1.13 \times 10^{-2}$ & $5.1 \times 10^{-2}$ \\
\hline & & & 54 & 2000 & 95 & 128 & 192 & 11 & 3 & $1.15 \times 10^{-2}$ & $5.0 \times 10^{-2}$ \\
\hline & & & 72 & 1000 & 95 & 128 & 192 & 11 & 3 & $1.11 \times 10^{-2}$ & $5.2 \times 10^{-2}$ \\
\hline & & & 90 & 1000 & 95 & 128 & 192 & 13 & 3 & $1.11 \times 10^{-2}$ & $5.9 \times 10^{-2}$ \\
\hline \multirow[t]{11}{*}{ DNS } & $10^{9}$ & 1 & 0 & 420 & 384 & 512 & 768 & 16 & 11 & $5.2 \times 10^{-3}$ & $7.8 \times 10^{-3}$ \\
\hline & & & 9 & 385 & 384 & 512 & 768 & 15 & 10 & $5.0 \times 10^{-3}$ & $7.7 \times 10^{-3}$ \\
\hline & & & 18 & 403 & 384 & 512 & 768 & 15 & 10 & $4.6 \times 10^{-3}$ & $7.6 \times 10^{-3}$ \\
\hline & & & 27 & 465 & 384 & 512 & 768 & 15 & 9 & $4.3 \times 10^{-3}$ & $7.6 \times 10^{-3}$ \\
\hline & & & 36 & 447 & 384 & 512 & 768 & 15 & 8 & $3.9 \times 10^{-3}$ & $7.6 \times 10^{-3}$ \\
\hline & & & 45 & 356 & 384 & 512 & 768 & 15 & 7 & $3.6 \times 10^{-3}$ & $7.5 \times 10^{-3}$ \\
\hline & & & 54 & 366 & 384 & 512 & 768 & 15 & 7 & $3.2 \times 10^{-3}$ & $7.4 \times 10^{-3}$ \\
\hline & & & 63 & 382 & 384 & 512 & 768 & 15 & 6 & $2.9 \times 10^{-3}$ & $7.7 \times 10^{-3}$ \\
\hline & & & 72 & 448 & 384 & 512 & 768 & 16 & 6 & $2.8 \times 10^{-3}$ & $8.0 \times 10^{-3}$ \\
\hline & & & 81 & 182 & 384 & 512 & 768 & 15 & 6 & $2.7 \times 10^{-3}$ & $8.4 \times 10^{-3}$ \\
\hline & & & 90 & 36 & 384 & 512 & 768 & 17 & 6 & $2.6 \times 10^{-3}$ & $8.7 \times 10^{-3}$ \\
\hline
\end{tabular}

TABLE 2. Details on the conducted DNS and LES, including the time of statistical averaging, $t_{\text {avg }}$, normalised with the free-fall time $t_{f}$; number of nodes $N_{r}, N_{\phi}, N_{z}$ in the directions $r, \phi$ and $z$, respectively; the number of the nodes within the thermal boundary layer, $\mathcal{N}_{t h}$, and within the viscous boundary layer, $\mathcal{N}_{v}$, and the relative thickness of the viscous boundary layer $\delta_{u} / L(2.17)$ and the thermal boundary layer $\delta_{t h} / L(2.16)$.

\subsection{Large-eddy simulations}

At any fixed time slice, LES generally require more computational effort per computational node, than the DNS. However, since the LES are relieved from the requirement to resolve the spatial Kolmogorov microscales, one can use significantly coarser meshes in the LES compared to those in the DNS, as soon as the LES are verified against the measurements from the physical point of view and against the DNS from the numerical point of view. Thus, the verified LES open the possibility 
of obtaining reliable data faster, compared to the DNS, using modest computational resources.

In our study, the OB equations (2.5)-(2.7) of thermogravitational convection with the LES approach for small-scale turbulence modelling are solved numerically using the open-source package OpenFOAM 4.1 (Weller et al. 1998) for $\operatorname{Pr}=0.0093$ and $R a=1.5 \times 10^{7}$ and 10 different inclination angles, equidistantly distributed between $\beta=0^{\circ}$ and $\beta=90^{\circ}$.

The package is configured as follows. The used LES model is that by SmagorinskyLilly (Deardorff 1970) with the Smagorinsky constant $C_{s}=0.17$, which is compatible with the value of the Kolmogorov spectrum constant for the inertial subrange. The turbulent Prandtl number in the core part of the domain equals $P r_{t}=0.9$ and smoothly vanishes close to the rigid walls. According to different models for liquid metals, the $\mathrm{Pr}_{t}$ value can be higher than this one (Chen et al. 2013). To estimate the effect of the $\operatorname{Pr}_{t}$ value on the simulation results we carried out additional LES with $\operatorname{Pr}_{t}$ values up to 4.12 and showed that the specific choice of $P r_{t}$ does not significantly affect the results. The utilised finite-volume solver is buoyantBoussinesqPimpleFoam with the pressure implicit with splitting of operators (PISO) algorithm by Issa (1986). Time integration is realised with the implicit Euler scheme; the diffusive and convective terms are also treated linearly (more precisely, using the filteredLinear scheme). The resulting systems of linear equations are solved with the preconditioned conjugate gradient method with the diagonal-based incomplete Cholesky preconditioner for the pressure and preconditioned biconjugate gradient method with the diagonal-based incomplete lower-upper (LU) preconditioner for other flow components (Fletcher 1976; Ferziger \& Perić 2002).

All simulations are carried out on a collocated non-equidistant computational grid consisting of 2.9 million nodes (see table 2). The grid has a higher density of nodes near the boundaries, in order to resolve the boundary layers. Further details on the numerical method, construction of the computational grid and model verification can be found in Mandrykin \& Teimurazov (2019).

\subsection{Methods of data analysis}

\subsubsection{Nusselt number and Reynolds number}

The main response characteristics of the convective system are the global heat and momentum transport represented by the dimensionless Nusselt number $N u$ and Reynolds number $R e$, respectively. Within the OB approximation, the Nusselt number equals

$$
N u=\left\langle\Omega_{z}\right\rangle_{z},
$$

where $\Omega_{z}$ is a component of the heat-flux vector along the cylinder axis:

$$
\Omega_{z} \equiv \frac{u_{z} T-\kappa \partial_{z} T}{\kappa \Delta / L}
$$

and $\langle\cdot\rangle_{z}$ denotes the average in time and over a cross-section at any distance $z$ from the heated plate.

The Reynolds number can be defined in different ways and one of the common definitions is based on the total kinetic energy of the system

$$
\operatorname{Re} \equiv(L / v) \sqrt{\langle\boldsymbol{u} \cdot \boldsymbol{u}\rangle} .
$$


Here, $\langle\cdot\rangle$ denotes the averaging in time and over the entire volume. We consider also the large-scale Reynolds number

$$
\operatorname{Re}_{U} \equiv(L / v) \sqrt{\left\langle\langle\boldsymbol{u}\rangle_{t}^{2}\right\rangle_{V}}
$$

where $\langle\cdot\rangle_{t}$ denotes the averaging in time and $\langle\cdot\rangle_{V}$ the averaging over the whole convection cell. Following Teimurazov \& Frick (2017), we also evaluate the Reynolds number based on the volume-averaged velocity fluctuations, or small-scale Reynolds number, as

$$
\operatorname{Re}_{u^{\prime}} \equiv(L / v) \sqrt{\left\langle\left(\boldsymbol{u}-\langle\boldsymbol{u}\rangle_{t}\right)^{2}\right\rangle} .
$$

Finally, one can calculate the Reynolds number based on the 'wind of turbulence' as follows:

$$
R e_{w}=(L / v) \max _{z} U_{w}(z),
$$

where the velocity of the wind, which is parallel to the heated or cooled plates, can be estimated by

$$
U_{w}(z)=\sqrt{\left\langle u_{\phi}^{2}+u_{r}^{2}\right\rangle_{z}},
$$

where $u_{\phi}$ and $u_{r}$ are the azimuthal and radial components of the velocity, respectively.

In the experiments, the Reynolds number is evaluated based on the average of the estimated axial velocities between the probes along the positions $\mathrm{A}$ and $\mathrm{E}$. The velocities are estimated as written below in $\$ 2.4 .4$.

\subsubsection{Boundary-layer thicknesses}

Close to the heated and cooled plates, thermal and viscous boundary layers develop. The thickness of the thermal boundary layer is calculated as

$$
\delta_{t h}=L /(2 N u) .
$$

This is the standard way to define the thickness of the thermal boundary layer under the assumption of pure conductive heat transport within this layer, cf. Ahlers et al. (2009).

Using the slope method (cf. Zhou \& Xia 2010), from the $U_{w}(z)$ profile along the cylinder axis, see (2.15), the viscous boundary-layer thickness $\delta_{u}$ is defined as follows:

$$
\delta_{u} \equiv \max _{z}\left\{U_{w}(z)\right\}\left(\left.\frac{\mathrm{d} U_{w}}{\mathrm{~d} z}\right|_{z=0}\right)^{-1} .
$$

The same criterion was used also for vertical convection before in $\mathrm{Ng}$ et al. (2015), and can also be adopted to the general case of IC.

\subsubsection{Properties of the large-scale circulation}

In the DNS and LES, the information on all flow components is available at every small finite volume associated with any grid node. In the experiments, all the information about the flow structures is obtained from the 28 temperature probes located as shown in figure 2 and discussed in $\S 2.1$. The probes are placed in five different horizontal cross-sections of the cylindrical sample, which are parallel to the heated or cooled surfaces. To make a comparison between the DNS, LES and the experiment possible, we measure the temperature and monitor its temporal evolution 


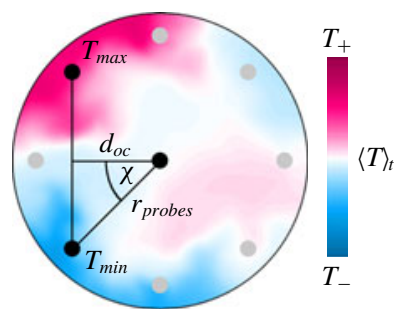

FIGURE 3. Sketch to illustrate the off-centre distance $d_{o c}$ of the sloshing mode, as obtained by the temperature extrema extraction method (cf. (2.19)), see Xi et al. (2009).

at the same locations in all three approaches. The only difference is that in the DNS and LES there are eight virtual probes in each cross-section, while in the experiment, there are eight probes in the cross-sections 1, 3 and 5 and only two probes in the cross-sections 2 and 4. The azimuthal locations $\mathrm{A}$ to $\mathrm{H}$ in the experiment, DNS and LES are exactly the same.

From the temperature measurements at the above discussed locations, one can evaluate the phase and the strength of the so-called wind of turbulence, or large-scale circulation (LSC). To do so, the method by Cioni et al. (1997) is applied, which is widely used in RBC experiments (Brown \& Ahlers 2006; Xi \& Xia 2007; Bai, Ji \& Brown 2016; He, Bodenschatz \& Ahlers 2016; Khalilov et al. 2018) and simulations (Mishra et al. 2011; Stevens, Clercx \& Lohse 2011; Wagner, Shishkina \& Wagner 2012; Ching, Dung \& Shishkina 2017). Thus, the temperature measured at 8 locations in the central cross-section, from $\mathrm{A} 3$ to $\mathrm{H} 3$ along the central circle 3 , is fitted by the cosine function

$$
T(\theta)=T_{m}+\delta_{3} \cos \left(\theta-\theta_{3}\right)
$$

to obtain the orientation of the LSC, represented by the phase $\theta_{3}$, and the strength of the first temperature mode, i.e. the amplitude $\delta_{3}$, which indicates the temperature drop between the opposite sides of the cylinder sidewall. At the warmer part of the sidewall, the LSC carries the warm plumes from the heated plate towards the cold plate and on the opposite colder part, it carries the cold plumes in the opposite direction.

In a similar way one can evaluate the LSC phases $\theta_{1}$ and $\theta_{5}$ and the strengths $\delta_{1}$ and $\delta_{5}$ at other heights from the heated plates, i.e. along circle 1 (closer to the heated plate) and along circle 5 (closer to the cooled plate), respectively.

To extract the sloshing mode we use the method by Xi et al. (2009). The so-called temperature extrema extraction (TEE) method is executed as follows: first, the maximum and minimum of the eight probes at a certain time and horizontal level are determined and then a second-order polynomial is fitted exactly through that maximum/minimum using one neighbouring probe to each side (3 points in total per fit). The off-centre distance of the sloshing mode is defined as

$$
d_{o c}=r_{\text {probes }} \cos (\chi)
$$

and it measures the shortest distance between the connecting line (of maximum and minimum temperature) and the centreline of the cylinder (cf. figure 3).

\subsubsection{Velocity estimates}

While in the DNS and LES the spatial distributions of all velocity components are available, direct measurements of the velocity in experiments on natural thermal 
convection in liquid sodium remain impossible so far. In order to estimate the velocities from the temperature measurements in the experiment, the cross-correlations for all combinations of any two neighbouring probes along the azimuthal locations from $\mathrm{A}$ to $\mathrm{H}$ are used.

For example, the normalised cross-correlation function $\left.C\right|_{A 1, A 2}(\tau)$ for the temporal dependences of the temperatures $\left.T\right|_{A 1}$ and $\left.T\right|_{A 2}$ measured by the probes at the locations $\mathrm{A} 1$ and $\mathrm{A} 2$ is calculated as follows:

$$
\left.C\right|_{A 1, A 2}(\tau) \propto \sum_{j}\left(\left.T\right|_{A 1}\left(t_{j}\right)-\left\langle\left. T\right|_{A 1}\right\rangle_{t}\right) \cdot\left(\left.T\right|_{A 2}\left(t_{j}+\tau\right)-\left\langle\left. T\right|_{A 2}\right\rangle_{t}\right) .
$$

The first maximum of the function $\left.C\right|_{A 1, A 2}(\tau)$ at $\tau=\tau_{c}$ provides the correlation time $\tau_{c}$. From the known distance between the probes A1 and A2 and the estimated time, $\tau_{c}$, which is needed for the flow to bring a thermal plume from the location A1 to A2, one can estimate the mean velocity of the flow between the locations A1 and A2.

In a similar way one estimates the mean velocities between the probes A2 and A3, etc., along the azimuthal location A. The mean velocities along the other azimuthal locations, from $\mathrm{B}$ to $\mathrm{H}$, are calculated analogously.

\section{Results and discussion}

In this section, we directly compare the results for inclined convection in a cylindrical container with a diameter-to-height aspect ratio of 1 , as they were obtained in the liquid-sodium DNS for $R a=1.67 \times 10^{7}$ and $\operatorname{Pr}=0.0094$, LES for $R a=1.5 \times 10^{7}$ and $\operatorname{Pr}=0.0093$ and liquid-sodium experiments for $R a=1.42 \times 10^{7}$ $(P r \approx 0.0093)$; see tables 3 and 4 .

Further liquid-sodium experiments were conducted to measure the scaling relations of the Nusselt number versus the Rayleigh number in the RBC (the inclination angle $\left.\beta=0^{\circ}\right)$ and $\mathrm{VC}$ configurations $\left(\beta=90^{\circ}\right)$, for the $R a$-range around $R a=10^{7}$.

Additionally, we make a comparison with the auxiliary DNS results for $R a=1.67 \times$ $10^{6}$ and $\operatorname{Pr}=0.094$, where the product of the Rayleigh number and Prandtl number, $\operatorname{RaPr} \approx 1.57 \times 10^{5}$, is the same as in the main DNS for liquid sodium with $R a=$ $1.67 \times 10^{7}$ and $\operatorname{Pr}=0.0094$ (see tables 3 and 4 ). This auxiliary case is interesting for the following reasons. The ratio of the thermal diffusion time scale, $t_{\kappa}=R^{2} / \kappa$, to the free-fall time scale, $t_{f}=\sqrt{R / \alpha g \Delta}$ is in both cases the same, since $t_{\kappa} / t_{f} \sim \sqrt{\operatorname{RaPr}}$. In contrast, in this auxiliary DNS case, the ratio $t_{v} / t_{f}$ of the viscous diffusion time scale $t_{v}=R^{2} / v$ to the free-fall time scale $t_{f}$ is approximately tenfold smaller than that in the main liquid-sodium DNS, albeit being approximately tenfold larger than $t_{\kappa} / t_{f}$. Hence, thermal diffusion dominates over viscous diffusion in both considered sets of parameters and for similar $\mathrm{RaPr}$ one might expect similar global temperature distributions and quantitatively similar heat and momentum transport in IC. Note that in the liquid-sodium DNS, the diffusion times are $t_{\kappa} \approx 140 t_{f}$ and $t_{v} \approx 14900 t_{f}$.

Another set of auxiliary DNS of IC is conducted for $\operatorname{Ra}=10^{9}$ and $\operatorname{Pr}=1$ for comparison. In this case, the Grashof number, $G r \equiv \operatorname{Ra} / \operatorname{Pr}=10^{9}$, is similar to that in the main liquid-sodium case $\left(G r \approx 1.8 \times 10^{9}\right)$, but for this Prandtl-number-one case and the liquid-sodium case we generally do not expect a close similarity of the global flow characteristics.

A summary of the conducted simulations and experiments can be found in table 2 . The free-fall time in the experiments equals $t_{f}=\sqrt{R /(\alpha g \Delta)} \approx 1.3 \mathrm{~s}$ and is similar to that in the main DNS and LES. Thus, the conducted DNS cover only approximately 


\begin{tabular}{|c|c|c|c|c|c|c|c|c|}
\hline & $R a$ & $\mathrm{Pr}$ & $\beta$ (deg.) & $\mathrm{Nu}$ & $R a$ & $P r$ & $\beta$ (deg.) & $N u$ \\
\hline \multirow[t]{14}{*}{ Exp. } & $5.27 \times 10^{6}$ & 0.0094 & 0 & 4.97 & $1.42 \times 10^{7}$ & 0.0093 & 20 & 6.51 \\
\hline & $6.43 \times 10^{6}$ & 0.0097 & 0 & 5.18 & $1.42 \times 10^{7}$ & 0.0093 & 30 & 7.02 \\
\hline & $9.32 \times 10^{6}$ & 0.0096 & 0 & 5.53 & $1.42 \times 10^{7}$ & 0.0093 & 40 & 7.07 \\
\hline & $1.12 \times 10^{7}$ & 0.0095 & 0 & 5.75 & $1.42 \times 10^{7}$ & 0.0093 & 50 & 7.15 \\
\hline & $1.18 \times 10^{7}$ & 0.0093 & 0 & 5.84 & $1.42 \times 10^{7}$ & 0.0093 & 60 & 7.23 \\
\hline & $1.28 \times 10^{7}$ & 0.0094 & 0 & 5.91 & $1.42 \times 10^{7}$ & 0.0093 & 70 & 7.30 \\
\hline & $1.42 \times 10^{7}$ & 0.0093 & 0 & 6.04 & $1.42 \times 10^{7}$ & 0.0093 & 80 & 7.13 \\
\hline & $1.43 \times 10^{7}$ & 0.0091 & 0 & 6.09 & $6.52 \times 10^{6}$ & 0.0095 & 90 & 5.87 \\
\hline & $1.55 \times 10^{7}$ & 0.0093 & 0 & 6.18 & $8.91 \times 10^{6}$ & 0.0094 & 90 & 6.19 \\
\hline & $1.80 \times 10^{7}$ & 0.0091 & 0 & 6.39 & $1.11 \times 10^{7}$ & 0.0093 & 90 & 6.53 \\
\hline & $2.06 \times 10^{7}$ & 0.0088 & 0 & 6.79 & $1.32 \times 10^{7}$ & 0.0091 & 90 & 6.77 \\
\hline & $2.18 \times 10^{7}$ & 0.0088 & 0 & 6.55 & $1.42 \times 10^{7}$ & 0.0093 & 90 & 6.84 \\
\hline & $2.37 \times 10^{7}$ & 0.0086 & 0 & 6.92 & $1.60 \times 10^{7}$ & 0.0090 & 90 & 7.07 \\
\hline & $1.42 \times 10^{7}$ & 0.0093 & 10 & 6.17 & $1.88 \times 10^{7}$ & 0.0086 & 90 & 7.47 \\
\hline \multirow[t]{2}{*}{ DNS } & $1.67 \times 10^{7}$ & 0.0094 & 0 & 9.59 & $1.67 \times 10^{7}$ & 0.0094 & 72 & 11.89 \\
\hline & $1.67 \times 10^{7}$ & 0.0094 & 36 & 12.24 & $1.67 \times 10^{7}$ & 0.0094 & 90 & 10.37 \\
\hline \multirow[t]{5}{*}{ LES } & $1.5 \times 10^{7}$ & 0.0093 & 0 & 9.27 & $1.5 \times 10^{7}$ & 0.0093 & 50 & 11.95 \\
\hline & $1.5 \times 10^{7}$ & 0.0093 & 10 & 9.65 & $1.5 \times 10^{7}$ & 0.0093 & 60 & 11.80 \\
\hline & $1.5 \times 10^{7}$ & 0.0093 & 20 & 10.28 & $1.5 \times 10^{7}$ & 0.0093 & 70 & 11.61 \\
\hline & $1.5 \times 10^{7}$ & 0.0093 & 30 & 11.41 & $1.5 \times 10^{7}$ & 0.0093 & 80 & 10.97 \\
\hline & $1.5 \times 10^{7}$ & 0.0093 & 40 & 11.71 & $1.5 \times 10^{7}$ & 0.0093 & 90 & 10.06 \\
\hline \multirow[t]{4}{*}{ DNS } & $1.67 \times 10^{6}$ & 0.0940 & 0 & 8.21 & $1.67 \times 10^{6}$ & 0.0940 & 36 & 9.79 \\
\hline & $1.67 \times 10^{6}$ & 0.0940 & 9 & 8.78 & $1.67 \times 10^{6}$ & 0.0940 & 54 & 9.95 \\
\hline & $1.67 \times 10^{6}$ & 0.0940 & 18 & 9.23 & $1.67 \times 10^{6}$ & 0.0940 & 72 & 9.55 \\
\hline & $1.67 \times 10^{6}$ & 0.0940 & 27 & 9.64 & $1.67 \times 10^{6}$ & 0.0940 & 90 & 8.55 \\
\hline \multirow[t]{6}{*}{ DNS } & $10^{9}$ & 1 & 0 & 63.74 & $10^{9}$ & 1 & 54 & 67.24 \\
\hline & $10^{9}$ & 1 & 9 & 64.58 & $10^{9}$ & 1 & 63 & 64.78 \\
\hline & $10^{9}$ & 1 & 18 & 65.81 & $10^{9}$ & 1 & 72 & 62.53 \\
\hline & $10^{9}$ & 1 & 27 & 65.58 & $10^{9}$ & 1 & 54 & 59.60 \\
\hline & $10^{9}$ & 1 & 36 & 66.05 & $10^{9}$ & 1 & 90 & 57.52 \\
\hline & $10^{9}$ & 1 & 45 & 67.15 & & & & \\
\hline
\end{tabular}

TABLE 3. Nusselt numbers, as they were obtained in the experiments, DNS and LES. The uncertainty in the experiments is approximately $3 \%$, in the DNS it is approximately $4 \%$ and IN the LES it is below $1 \%$.

two minutes of the real-time experiment, which was conducted for approximately $7 \mathrm{~h}$. One should note that although the DNS statistical averaging time is quite short, collecting approximately $100 t_{f}$ statistics for the case $\beta=0^{\circ}$ consumed approximately 390000 CPUh at the SuperMUC at the Leibniz Supercomputing Centre and required approximately 60 days of runtime.

In the remaining part of this section we investigate integral time-averaged quantities such as the global heat transport (Nusselt number) and the global momentum transport (Reynolds number), provide the evidence of a quantitative agreement between the simulations and experiment and present a complementary picture of the dynamics of the large-scale flows in liquid-sodium IC. 


\begin{tabular}{|c|c|c|c|c|c|c|}
\hline & $R a$ & $P r$ & $\beta$ (deg.) & $R e$ & $R e_{u^{\prime}}$ & $R e_{U}$ \\
\hline DNS & $1.67 \times 10^{7}$ & 0.0094 & $\begin{array}{r}0 \\
36 \\
72 \\
90\end{array}$ & $\begin{array}{l}1.80 \times 10^{4} \\
1.95 \times 10^{4} \\
1.34 \times 10^{4} \\
1.01 \times 10^{4}\end{array}$ & $\begin{array}{r}1.27 \times 10^{4} \\
1.02 \times 10^{4} \\
5.5 \times 10^{3} \\
4.3 \times 10^{3}\end{array}$ & $\begin{array}{r}1.27 \times 10^{4} \\
1.66 \times 10^{4} \\
1.22 \times 10^{4} \\
9.1 \times 10^{3}\end{array}$ \\
\hline LES & $1.5 \times 10^{7}$ & 0.0093 & $\begin{array}{r}0 \\
10 \\
20 \\
30 \\
40 \\
50 \\
60 \\
70 \\
80 \\
90\end{array}$ & $\begin{array}{r}1.627 \times 10^{4} \\
1.702 \times 10^{4} \\
1.766 \times 10^{4} \\
1.772 \times 10^{4} \\
1.723 \times 10^{4} \\
1.676 \times 10^{4} \\
1.517 \times 10^{4} \\
1.311 \times 10^{4} \\
1.147 \times 10^{4} \\
9.60 \times 10^{3}\end{array}$ & $\begin{array}{r}1.136 \times 10^{4} \\
1.145 \times 10^{4} \\
1.113 \times 10^{4} \\
9.70 \times 10^{3} \\
8.24 \times 10^{3} \\
7.11 \times 10^{3} \\
6.02 \times 10^{3} \\
5.32 \times 10^{3} \\
4.60 \times 10^{3} \\
3.85 \times 10^{3}\end{array}$ & $\begin{array}{l}1.165 \times 10^{4} \\
1.258 \times 10^{4} \\
1.371 \times 10^{4} \\
1.483 \times 10^{4} \\
1.513 \times 10^{4} \\
1.518 \times 10^{4} \\
1.392 \times 10^{4} \\
1.199 \times 10^{4} \\
1.051 \times 10^{4} \\
8.80 \times 10^{3}\end{array}$ \\
\hline DNS & $1.67 \times 10^{6}$ & 0.0940 & $\begin{array}{r}0 \\
9 \\
18 \\
27 \\
36 \\
54 \\
72 \\
90\end{array}$ & $\begin{array}{r}1.33 \times 10^{3} \\
1.42 \times 10^{3} \\
1.46 \times 10^{3} \\
1.46 \times 10^{3} \\
1.43 \times 10^{3} \\
1.33 \times 10^{3} \\
9.9 \times 10^{2} \\
7.3 \times 10^{2}\end{array}$ & $\begin{array}{c}1.24 \times 10^{3} \\
1.10 \times 10^{3} \\
8.1 \times 10^{2} \\
7.0 \times 10^{2} \\
6.3 \times 10^{2} \\
3.7 \times 10^{2} \\
7.7 \times 10^{1} \\
0\end{array}$ & $\begin{array}{r}4.7 \times 10^{2} \\
1.10 \times 10^{3} \\
1.22 \times 10^{3} \\
1.28 \times 10^{3} \\
1.28 \times 10^{3} \\
1.28 \times 10^{3} \\
9.9 \times 10^{2} \\
7.3 \times 10^{2}\end{array}$ \\
\hline DNS & $10^{9}$ & 1 & $\begin{array}{r}0 \\
9 \\
18 \\
27 \\
36 \\
45 \\
54 \\
63 \\
72 \\
81 \\
90\end{array}$ & $\begin{array}{r}4.72 \times 10^{3} \\
5.13 \times 10^{3} \\
4.97 \times 10^{3} \\
4.65 \times 10^{3} \\
4.06 \times 10^{3} \\
3.79 \times 10^{3} \\
3.31 \times 10^{3} \\
2.44 \times 10^{3} \\
1.66 \times 10^{3} \\
1.23 \times 10^{3} \\
8.4 \times 10^{2}\end{array}$ & $\begin{array}{r}4.25 \times 10^{3} \\
3.62 \times 10^{3} \\
2.50 \times 10^{3} \\
2.14 \times 10^{3} \\
1.91 \times 10^{3} \\
1.84 \times 10^{3} \\
1.68 \times 10^{3} \\
1.29 \times 10^{3} \\
7.5 \times 10^{2} \\
2.9 \times 10^{2} \\
1.2 \times 10^{1}\end{array}$ & $\begin{array}{r}2.05 \times 10^{3} \\
3.63 \times 10^{3} \\
4.29 \times 10^{3} \\
4.12 \times 10^{3} \\
3.59 \times 10^{3} \\
3.31 \times 10^{3} \\
2.85 \times 10^{3} \\
2.07 \times 10^{3} \\
1.48 \times 10^{3} \\
1.19 \times 10^{3} \\
8.4 \times 10^{2}\end{array}$ \\
\hline
\end{tabular}

TABLE 4. Reynolds numbers, a they were obtained in the DNS and LES, see the definitions (2.11), (2.12) and (2.13). The numerical uncertainty of the Reynolds numbers was estimated to be approximately $3 \%$ for DNS and below $1 \%$ for LES.

\subsection{Time-averaged heat and momentum transport}

First, we examine the classical case of $\operatorname{RBC}$ without inclination $\left(\beta=0^{\circ}\right)$. The time-averaged mean heat fluxes, represented by the Nusselt numbers, are presented in figure 4.

There is an excellent agreement of the DNS and LES data, for example, the Nusselt number deviates less than $1 \%$ and the Reynolds number around $5 \%$ for the RBC and VC cases. Also in figure 4 we compare our numerical results with the DNS by Scheel \& Schumacher (2017), for $\operatorname{Pr}=0.005$ and $\operatorname{Pr}=0.025$. Our numerical results for $P r=0.0094$ and $P r=0.0093$ take place between the cited results by Scheel \& Schumacher (2017), as expected. Note that our LES and DNS and the DNS by Scheel 


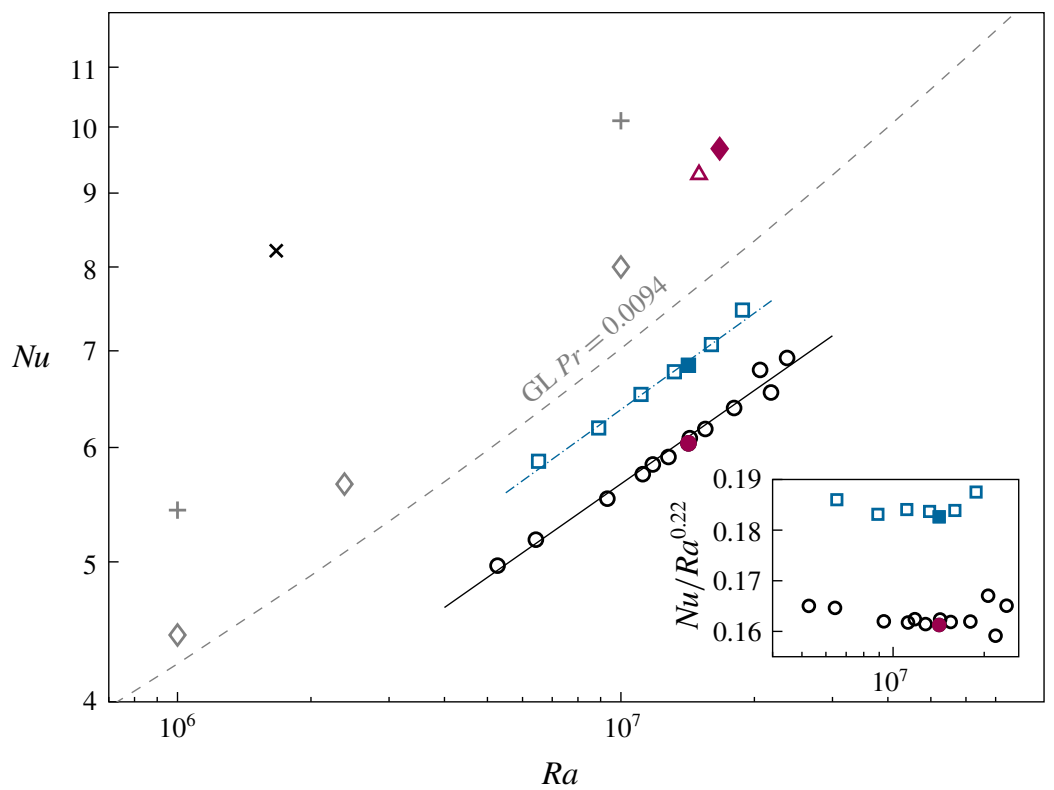

FIGURE 4. Nusselt number versus Rayleigh number, as obtained in the RBC experiments for different Rayleigh numbers, $\operatorname{Pr} \approx 0.009, \beta=0^{\circ}$ (open circles) with the effective scaling $N u \approx 0.177 R a^{0.215}$ (solid line); the RBC experiment for $R a=1.42 \times 10^{7}, P r=0.0093, \beta=$ $0^{\circ}$ (filled circle; this run is the longest one $(7 \mathrm{~h})$ in the series of measurements. For the same $R a$ and $P r$, the Nusselt numbers were also measured for different $\beta$, see table 3); the VC experiments for different Rayleigh numbers, $\operatorname{Pr} \approx 0.009, \beta=90^{\circ}$ (open squares) with the effective scaling $N u \approx 0.178 R a^{0.222}$ (dash-dotted line); the inset shows the compensated Nusselt number for RBC and VC experimental data; the DNS for $R a=1.67 \times 10^{7}, \operatorname{Pr}=$ 0.0094, $\beta=0^{\circ}$ (filled diamond); the DNS for $R a=1.67 \times 10^{6}, \operatorname{Pr}=0.094, \beta=0^{\circ}$ (cross); the LES for $R a=1.5 \times 10^{7}, \operatorname{Pr}=0.0093, \beta=0^{\circ}$ (open triangle). Results of the RBC DNS by Scheel \& Schumacher (2017) for $P r=0.005$ (open diamonds) and for $P r=0.025$ (pluses) and predictions for $P r=0.0094$ of the Grossmann \& Lohse $(2000,2001)$ theory considered with the pre-factors from Stevens et al. (2013) (dash line) are presented for comparison. Everywhere a cylindrical convection cell of aspect ratio 1 is considered.

\& Schumacher (2017) were conducted using completely different codes (the Nek5000 spectral element package in the latter case), but nevertheless lead to consistent results. This verifies to the fact that the obtained results are independent of the numerical method.

The experimental data exhibit generally lower Nusselt numbers (approximately $35 \%$ ) compared to the numerical data and this can be explained by the following two reasons. First, the ideal boundary conditions of constant temperatures at the plates cannot be provided in the experiments, since each emission of a sufficiently strong thermal plume affects, at least for a short time, the local temperature at the plate, which results in a reduction of the averaged heat flux compared to that in the simulations with the ideal boundary conditions. Second, the impossibility of measuring the temperature directly at the outer surfaces of the copper plates leads to a slight overestimation of $\Theta$ and $\Delta$ (see figure 1) and, hence, of the effective Rayleigh numbers for the measured Nusselt numbers. 


\subsubsection{Scaling relations}

For $\operatorname{Pr}=0.0094$, the predictions by the Grossmann \& Lohse $(2000,2001)$ theory, with the prefactors from Stevens et al. (2013), are shown in figure 4 between the obtained experimental and numerical data. The experimental data for a certain $R a-$ range around $R a=10^{7}$, shown in figure 4, follow a scaling relation

$$
N u \approx(0.16 \pm 0.01) R a^{0.22 \pm 0.06} \quad\left(\mathrm{RBC}, \beta=0^{\circ}\right)
$$

The fitted scaling exponent $\gamma$ for the RBC case is smaller than the scaling exponents measured in liquid gallium $(\gamma=0.249$, King \& Aurnou (2013)) and liquid mercury $(\gamma=0.285$, Takeshita et al. (1996)). The Prandtl number for both fluids is $P r \approx 0.025$.

Horanyi et al. (1999) measured the heat transport in liquid sodium at $\operatorname{Pr} \approx 0.006$ for $R a \leqslant 4.5 \times 10^{6}$. They used larger aspect ratios from 4.5 to 20 , while their highest $R a$ measurements were conducted in cells with the smallest aspect ratio. Nevertheless, their $N u \approx 5$ at their highest $R a \approx 4.5 \times 10^{6}$ is similar to $N u=4.97$ at our lowest $R a=5.27 \times 10^{6}$. Furthermore, Horanyi et al. (1999) found that $N u$ scales as $R a^{0.25}$, which agrees with our results.

The GL theory predicts a local scaling exponent ranging from $0.23\left(R a=5 \times 10^{6}\right)$ to $0.26\left(R a=3 \times 10^{7}\right)$ for $\operatorname{Pr}=0.0094$. This results in a mean scaling exponent of approximately 0.25 for this interval of Rayleigh numbers.

We fit $\log (N u)$ versus $\log (R a)$ with a second-order polynomial to estimate the uncertainty of the scaling exponent in our experiments. The local scaling exponent of this fit ranges from $0.16\left(R a=5 \times 10^{6}\right)$ to $0.27\left(R a=3 \times 10^{7}\right)$, thus, we estimate the uncertainty of the scaling exponent to be $\Delta \gamma= \pm 0.06$. A large variation of the local scaling exponent can be seen in Glazier et al. (1999) for liquid mercury in an aspect ratio one cylinder. Their local scaling exponent for small Rayleigh numbers $\left(\sim 10^{6}-10^{8}\right)$ is 0.25 , while their average scaling exponent is 0.285 . Glazier et al. (1999) argue that the 'strong steady bulk mean flow in the cell of aspect ratio one' reduces the scaling exponent. Presumably, we see a similar effect in our measurements.

In figure 4 we also present the measured scaling relations for $N u$ versus $R a$ for the case of $\mathrm{VC}\left(\beta=90^{\circ}\right)$. The scaling relation in $\mathrm{VC}$ is found to be quite similar to that in $\mathrm{RBC}$, namely

$$
N u \approx(0.18 \pm 0.01) R a^{0.22 \pm 0.06} \quad\left(\mathrm{VC}, \beta=90^{\circ}\right)
$$

The absolute values of the Nusselt numbers in VC are, however, larger than in RBC.

\subsubsection{Time-averaged heat transport in inclined convection}

In figure $5(a)$, the Nusselt numbers in IC are presented, which are normalised by $N u$ of the RBC case, for the same $R a$ and $P r$, i.e. the dependence of $N u(\beta) / N u\left(0^{\circ}\right)$ on the inclination angle $\beta$. Very remarkable is that, for similar $R a$ and $P r$, the DNS and LES deliver very similar values of $N u$. As previously discussed for the limiting cases (RBC and VC), the deviation between DNS and LES data for the Nusselt numbers is only a few per cent. One can see that the relative increase $N u(\beta) / N u\left(0^{\circ}\right)$ of the numerical data is up to 10 percentage points higher than that of the experimental data for an inclination angle of approximately $50^{\circ}$. This is accompanied by a shift of the inclination angle with maximal heat transport from approximately $50^{\circ}$ in our numerical results to $70^{\circ}$ in our experimental results. On the one hand, one may take into account 

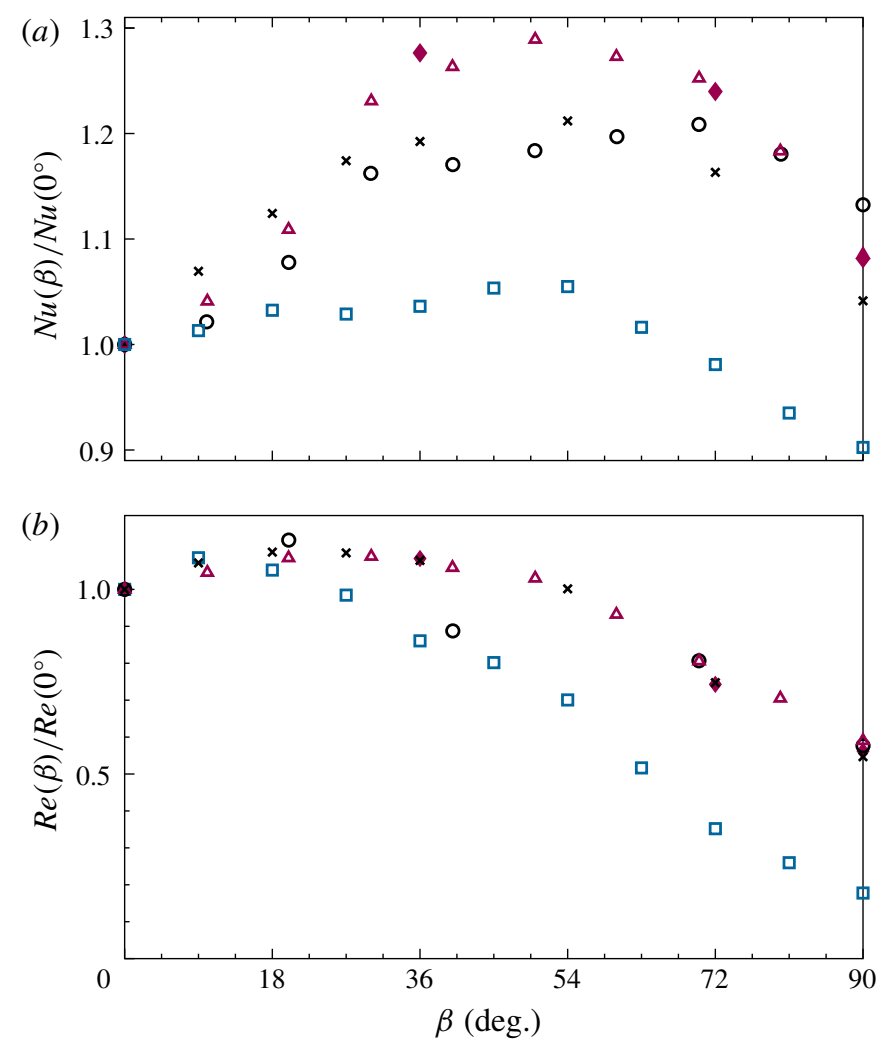

FIgURE 5. (a) Normalised Nusselt number $N u(\beta) / N u\left(0^{\circ}\right)$ versus the inclination angle $\beta$, as obtained in the DNS for $R a=1.67 \times 10^{7}, \operatorname{Pr}=0.0094$ (filled diamonds), the LES for $R a=1.5 \times 10^{7}, \operatorname{Pr}=0.0093$ (open triangles), the DNS for $R a=1.67 \times 10^{6}, \operatorname{Pr}=0.094$ (crosses), the experiments for $R a=1.42 \times 10^{7}, \operatorname{Pr} \approx 0.0093$ (open circles) and the DNS for $R a=10^{9}, \operatorname{Pr}=1$ (squares). (b) Normalised Reynolds number versus the inclination angle $\beta$, as obtained in the same DNS and LES as in $(a)$; similar symbols are used as in $(a)$.

that the Rayleigh number in the experiment is slightly smaller $\left(R a=1.42 \times 10^{7}\right)$ compared to that in the DNS $\left(R a=1.67 \times 10^{7}\right)$ and LES $\left(R a=1.5 \times 10^{7}\right)$. On the other hand, the heat transport in the inclined case is enhanced up to $29 \%$ which might reduce the heat loss $Q_{\text {loss }}$. However, $Q_{\text {loss }}$ is measured in the isothermal case, maintaining the mean temperature in the cylinder (cf. \$2.1). If the heat loss in the inclined case is less than in the isothermal reference case, then also the calculated effective power $Q_{\text {eff }}=Q-Q_{\text {loss }}$ is lower, and so is the Nusselt number.

As discussed above, we want to compare our results for liquid sodium with the DNS data for similar $R a P r$ and with the DNS data for similar $R a / P r$, as in our liquidsodium measurements and numerical simulations. Figure $5(a)$ and table 3 show that the obtained Nusselt numbers for the same $\operatorname{Ra} \operatorname{Pr}\left(\operatorname{Ra}=1.67 \times 10^{6}, \operatorname{Pr}=0.094\right)$ are in good agreement (the difference is less than 10 percentage points) with the liquidsodium experimental results $\left(\operatorname{Ra}=1.42 \times 10^{7}, \operatorname{Pr} \approx 0.0093\right)$. Remarkable is that, not only the relative Nusselt number, $N u(\beta) / N u\left(0^{\circ}\right)$, but also the absolute values of $N u$ are very similar in the liquid-sodium case and in the case of different $\operatorname{Pr}<1$ but the same $\mathrm{RaPr}$. In contrast to that, the $\mathrm{Nu}$-dependence on the inclination angle in the case of the same Grashof number is different, as expected. In that case, the maximal relative 
increase of the Nusselt number due to the cell inclination is only approximately $6 \%$, while in the liquid-sodium case it is up to $29 \%$.

\subsubsection{Time-averaged momentum transport in inclined convection}

In figure $5(b)$ and table 4 , the results for the Reynolds number are presented for the liquid-sodium measurements and simulations as well as for the auxiliary DNS. Again, the agreement between the experiments, DNS and LES for liquid sodium is excellent, usually less than $10 \%$ between DNS and LES. The dependences of $\operatorname{Re}(\beta) / \operatorname{Re}\left(0^{\circ}\right)$ on the inclination angle, obtained in the liquid-sodium DNS and in the DNS for the same product of $\operatorname{RaPr}$, demonstrate perfect agreement (less than two percentage points difference). The values of $\operatorname{Re}(\beta) / \operatorname{Re}\left(0^{\circ}\right)$ first slightly increase (around $10 \%$ ) with the inclination angle and then smoothly decrease, so that the Reynolds number $\operatorname{Re}\left(90^{\circ}\right)$ in the $\mathrm{VC}$ case is significantly smaller (only approximately $50 \%$ ) than the Reynolds number $\operatorname{Re}\left(0^{\circ}\right)$ in the $\mathrm{RBC}$ case. Here one should notice that the absolute values of $R e$ in the liquid-sodium case are significantly larger than in the IC flows for a similar $\mathrm{RaPr}$. In the case of the almost similar Grashof number, the Reynolds numbers decrease much faster with increasing inclination angle than in the liquidsodium case.

\subsubsection{Time-averaged flow structures in inclined convection}

Since the Nusselt numbers and relative Reynolds numbers behave very similarly in the liquid-sodium IC experiments and in the DNS for similar $R a P r$, we compare the time-averaged flow structures for these cases in figures 6 and 7. In these figures, the time-averaged temperature (figure 6) and the time-averaged component of the heat-flux vector parallel to the cylinder axis $\left\langle\Omega_{z}\right\rangle_{t}$ (figure 7) are presented in the plane of the LSC, for different inclination angles. One can see that both the temperature distributions and the heat-flux distributions, in the liquid-sodium case and in the case of a similar $R a P r$, look almost identical. In contrast to them, the corresponding distributions for a similar Grashof number look very different. The difference is especially pronounced for the inclination angle $\beta=36^{\circ}$. While in the liquid-sodium flow for $\beta=36^{\circ}$ there persist two intertwined plumes, a hot one and a cold one, the temperature in the Prandtl-number-one case is better mixed (figure 6) and the heat-flux distribution appears in a form of two triangular-shaped separated spots (figure 7).

From what is presented above, one can see that at almost similar Grashof numbers convection of a Prandtl-number-one fluid leads neither to similar integral quantities like $N u$ or $R e$, nor to similar heat flow structures in IC. Thus, it is clearly different to small-Prandtl-number IC flows. In contrast to that, the small-Prandtl-number IC flows of similar $\operatorname{Ra} \operatorname{Pr}$ have similar Nusselt numbers $N u$, similar relative Reynolds numbers $\operatorname{Re}(\beta) / \operatorname{Re}\left(0^{\circ}\right)$ and similar mean temperature and heat-flux distributions.

\subsection{Temperature and velocity profiles}

In this section we analyse the temperature and velocity profiles. The focus thereby is on the following two aspects. First, we compare the experimentally and numerically obtained profiles through the probes (positions A to $\mathrm{H}$ ) along the lines aligned parallel to the cylinder axes. Second, we compare the velocity profiles, obtained in the DNS and LES, with the velocities evaluated from the correlation times between two neighbouring probes in the experiment, in order to validate the method used in the experiment to estimate the Reynolds number. 


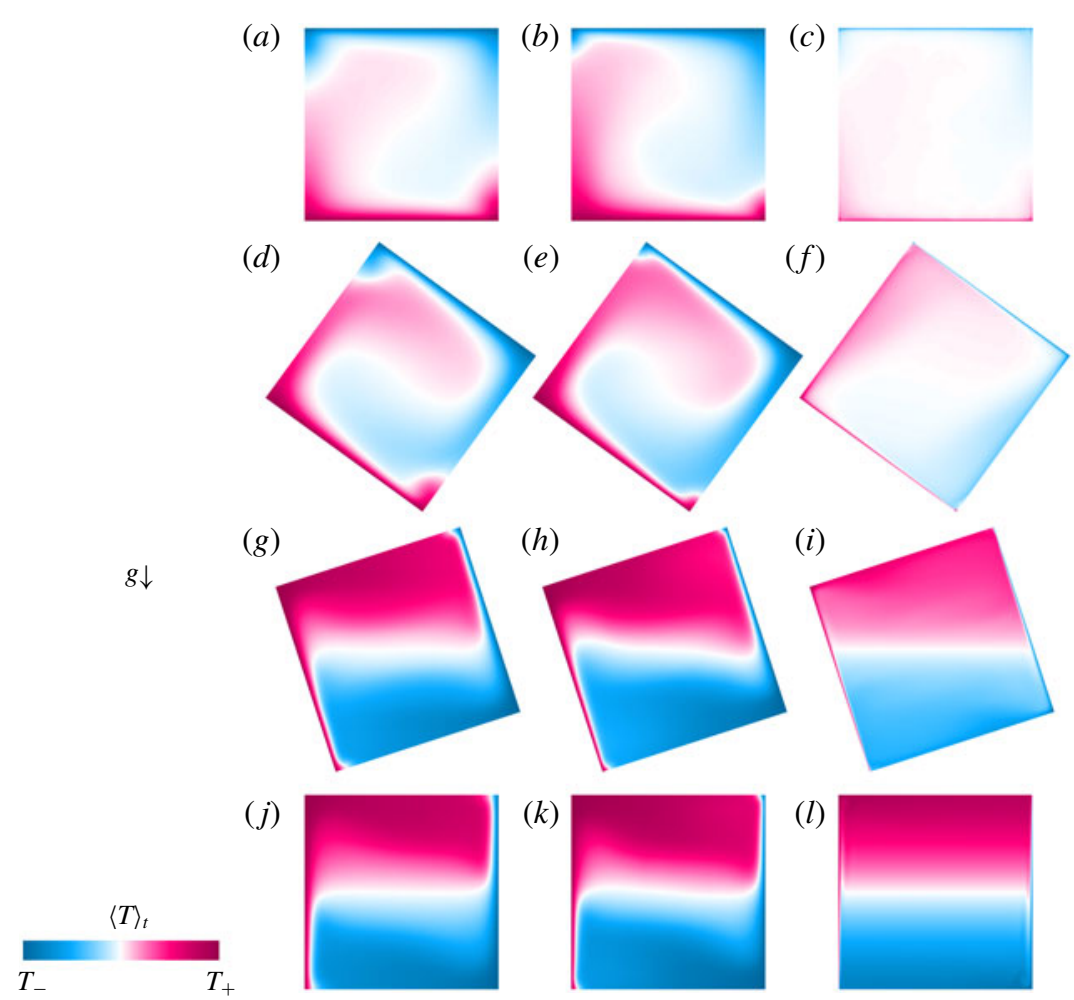

FIGURE 6. Table of vertical slices through the time-averaged temperature in the plane of the LSC, as obtained in the DNS for $R a=1.67 \times 10^{6}, \operatorname{Pr}=0.094(a, d, g, j) ; R a=1.67 \times 10^{7}$, $\operatorname{Pr}=0.0094(b, e, h, k)$ and $R a=10^{9}, \operatorname{Pr}=1(c, f, i, l)$. From $(a)$ to $(l)$, the inclination angle $\beta$ changes from $\beta=0^{\circ}$ (RBC case) through $\beta=36^{\circ}$ and $\beta=72^{\circ}$ to $\beta=90^{\circ}$ (VC case).

In figure $8(a)$, the time-averaged temperature profiles along the lines of the probe positions $\mathrm{A}$ to $\mathrm{H}$ are presented for the inclination angles $\beta=36^{\circ}$ (DNS) and $\beta=40^{\circ}$ (LES and experiments). Figure 8(b) shows analogous profiles for the inclination angles $\beta=72^{\circ}$ (DNS) and $\beta=70^{\circ}$ (LES and experiments). In both figures, the profiles at the positions $\mathrm{A}$ and $\mathrm{E}$ are presented, as well as the average of the profiles at the positions $\mathrm{B}$ and $\mathrm{H}$, the average of the profiles at the positions $\mathrm{D}$ and $\mathrm{F}$ and the average of the C-profile and G-profile. One can see that for the same locations, the LES and DNS profiles are almost indistinguishable, which again demonstrates quantitative agreement between the DNS and LES. The experimental data are available pointwise there, according to the 5 or 3 probes along each location, from A to $\mathrm{H}$. The measurements of the time-averaged temperatures are found to be in good agreement (largest deviation approximately $0.1 \Delta$ ) with the numerical data, taking into account that the Rayleigh number in the experiments is approximately $15 \%$ smaller than in the DNS.

At an inclination angle of approximately $\beta=36^{\circ}$ or $\beta=40^{\circ}$ (figure $8 a$ ), the mean temperature gradient with respect to the direction $z$ across the plates is close to zero in the core part of the domain. This means that the turbulent mixing in this case is very efficient, which is also reflected in the increased Nusselt numbers that we studied before. In contrast to that, for the inclination angle of approximately $\beta=70^{\circ}$ 
(a)

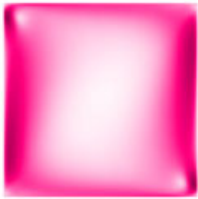

(d)

$g \downarrow$

(g)

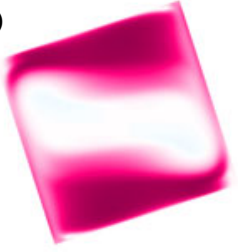

(j)

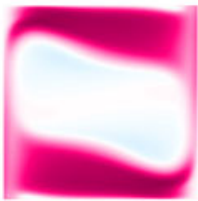

(b)

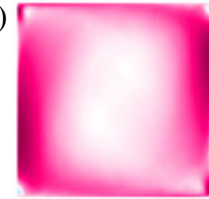

(e)

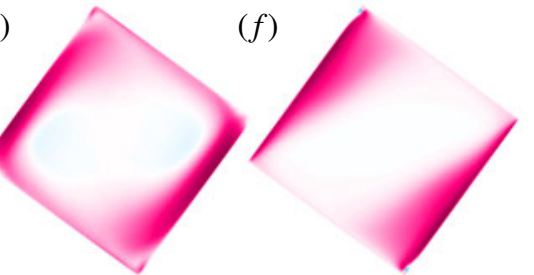

(h)

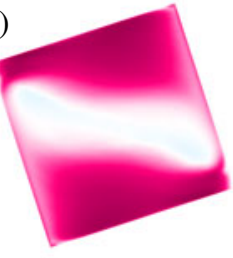

(k)

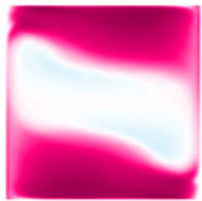

$(c)$

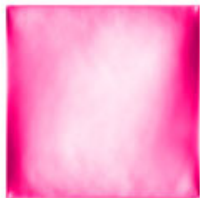

(i)

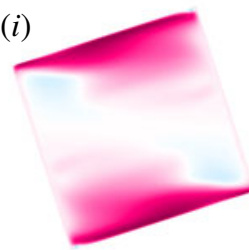

(l)

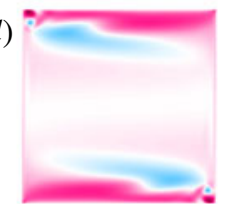

FIgURE 7. Table of vertical slices in the plane of the LSC of the time-averaged component of the heat-flux vector parallel to the cylinder axis, $\left\langle\Omega_{z}\right\rangle_{t}$, normalised by its maximal value through the entire volume, $\Omega_{\max }$, as obtained in the DNS for $R a=$ $1.67 \times 10^{6}, \operatorname{Pr}=0.094(a, d, g, j) ; R a=1.67 \times 10^{7}, \operatorname{Pr}=0.0094(b, e, h, k)$ and $R a=10^{9}$, $\operatorname{Pr}=1(c, f, i, l)$. From $(a)$ to $(l)$, the inclination angle $\beta$ changes from $\beta=0^{\circ}$ (RBC case) through $\beta=36^{\circ}$ and $\beta=72^{\circ}$ to $\beta=90^{\circ}$ (VC case).

(figure $8 b$ ), the mean flow is stratified and the temperature profiles have non-vanishing gradients in the $z$-direction.

In figure 9, the time-averaged profiles of the velocity component $u_{z}$ along the line of the probe positions $(\mathrm{A}-\mathrm{H})$ parallel to the cylinder axis are presented for the same inclinations angles, as in figure 8 . The velocity estimates at the locations between the neighbouring thermocouples, which are derived from the correlation times obtained in the temperature measurements, are found to be in agreement (largest deviation approximately $0.27 U_{f}$ ) with the DNS and LES data. Thus, this method to estimate the LSC velocity from the temperature measurement is proved to be a very reliable instrument in the IC liquid-sodium experiments.

The viscous boundary-layer thickness ((2.17) and table 2) for the liquid-sodium DNS and LES agree within a small deviation of approximately $3 \%$. With inclination, the viscous boundary-layer thickness first decreases slightly (by a few per cent) and then gradually increases. Note that the viscous boundary layers defined on the velocity magnitude (Ching et al. 2017) and on the wall shear stress (Scheel \& Schumacher 2016) are, respectively, approximately two and three times thicker than the boundary layer defined by (2.17) and table 2 . 

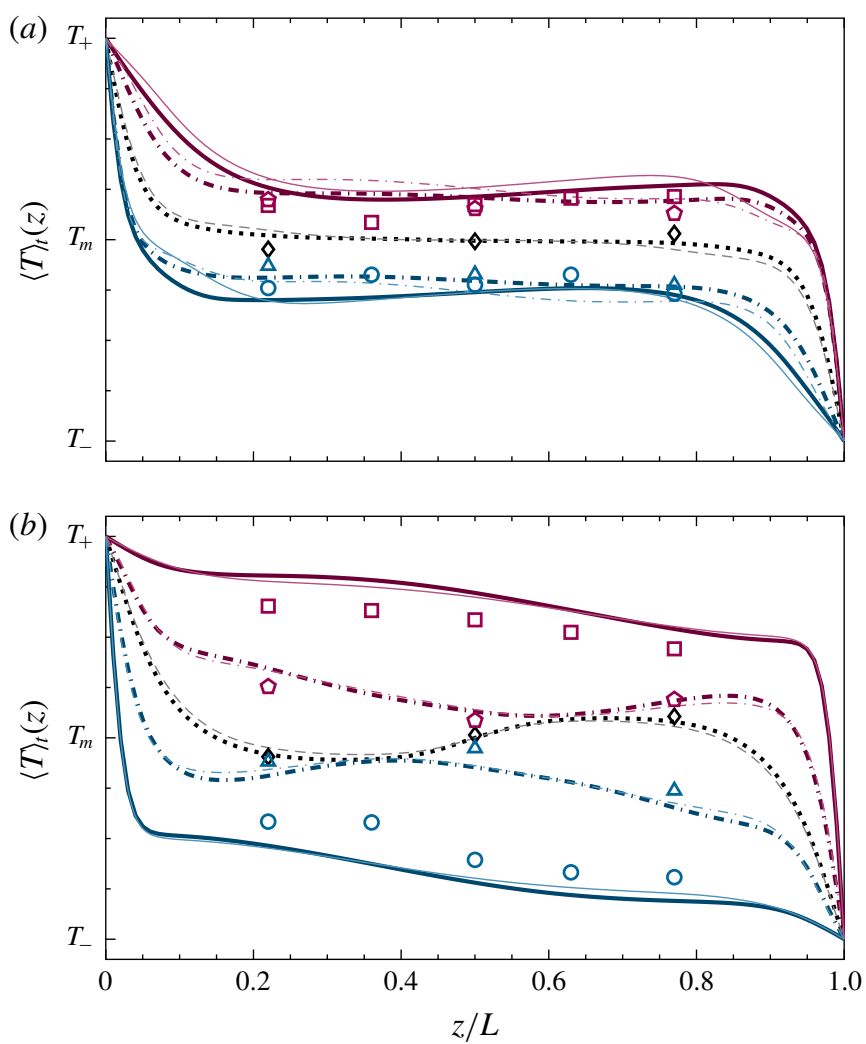

FIgURE 8. Time-averaged temperature profiles at the positions $\mathrm{A}$ to $\mathrm{H}$ of the probes, as obtained in $(a)$ the DNS for $\beta=36^{\circ}$ and the LES and experiments for $\beta=40^{\circ}$ and in (b) the DNS for $\beta=72^{\circ}$ and the LES and experiments for $\beta=70^{\circ}$. Thick lines are the DNS data, thin lines are the LES data and symbols are the experimental data. The DNS data are for $R a=1.67 \times 10^{7}, \operatorname{Pr}=0.0094$, the LES data are for $\operatorname{Ra}=1.5 \times 10^{7}, \operatorname{Pr}=0.0093$ and the experiments are for $R a=1.42 \times 10^{7}, \operatorname{Pr} \approx 0.0093$. Data at the position A (pink solid lines, squares) and the position $\mathrm{E}$ (blue solid lines, circles); the average of the data at the positions $\mathrm{B}$ and $\mathrm{H}$ (pink dash-dotted lines, pentagons), the average of the data at the positions $\mathrm{D}$ and $\mathrm{F}$ (blue dash-dotted lines, triangles) and the average of the data at the positions $\mathrm{C}$ and $\mathrm{G}$ (black dotted and grey dash lines, diamonds). The data are averaged along opposite positions due to the expected symmetry and in order to reduce the number of lines in the plot.

\subsection{Dynamics of the large-scale flow}

In this section, we focus on the reconstruction of the rich structural dynamics of the large-scale IC flows in liquid sodium.

It is well known from the previous RBC studies that the LSC in RBC can show different azimuthal orientations (Brown \& Ahlers 2006; Wagner et al. 2012) and can exhibit complicated dynamics with twisting (Funfschilling \& Ahlers 2004; Funfschilling, Brown \& Ahlers 2008; He et al. 2016) and sloshing (Xi, Zhou \& Xia 2006; Xi et al. 2009; Brown \& Ahlers 2009; Zhou et al. 2009; Bai et al. 2016; Zürner et al. 2019). In very-low-Prandtl-number RBC, this complicated behaviour of the LSC was reported in experiments with mercury by Cioni et al. (1997) and in the simulations by Schumacher et al. (2016) and Scheel \& Schumacher (2016, 2017). 

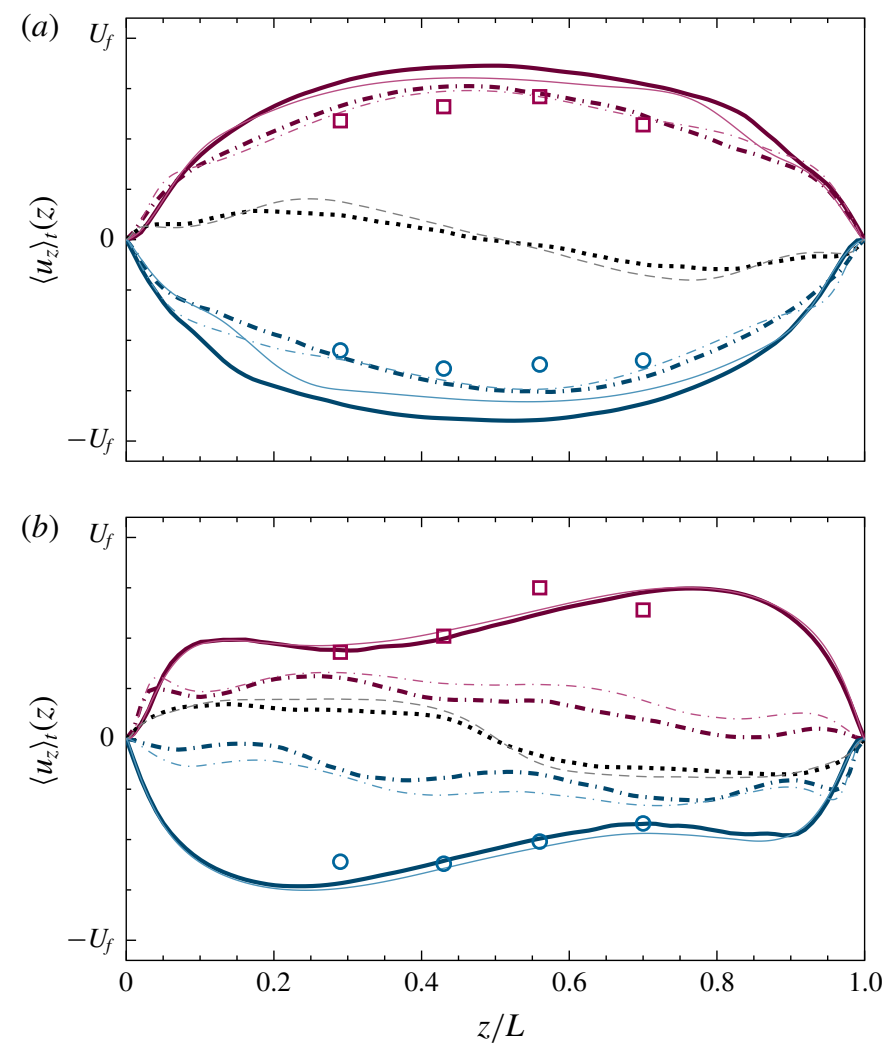

FIgURE 9. Time-averaged profiles of the velocity component $u_{z}$, which is parallel to the cylinder axis, considered at the positions $\mathrm{A}$ to $\mathrm{H}$ of the probes, as obtained in $(a)$ the DNS for $\beta=36^{\circ}$ and the LES and experiments for $\beta=40^{\circ}$ and in $(b)$ the DNS for $\beta=72^{\circ}$ and the LES and experiments for $\beta=70^{\circ}$. Thick lines are the DNS data, thin lines are the LES data and symbols are the experimental data. The DNS data are for $R a=1.67 \times 10^{7}$, $\operatorname{Pr}=0.0094$, the LES data are for $R a=1.5 \times 10^{7}, \operatorname{Pr}=0.0093$ and the experiments are for $R a=1.42 \times 10^{7}, P r \approx 0.0093$. Data at the position A (pink solid lines, squares) and the position $\mathrm{E}$ (blue solid lines, circles); the average of the data at the positions $\mathrm{B}$ and $\mathrm{H}$ (pink dash-dotted lines), the average of the data at the positions D and F (blue dash-dotted lines) and the average of the data at the positions $C$ and $G$ (black dotted and grey dash lines). The data are averaged along opposite positions due to the expected symmetry and in order to reduce the number of lines in the plot.

In our simulations and experiments in liquid sodium, we observe the twisting and sloshing dynamics of the LSC in the RBC configuration of the flow, i.e. without any cell inclination, as well as for small inclination angles $\beta$ until a certain critical $\beta=\beta_{s}$. The experimental data suggest that a transition to the non-twisting behaviour of the LSC is quite sharp and it is presumably caused by the increasing stratification of the temperature at larger inclination angles (Khalilov et al. 2018).

No LSC cessations were captured in the experiments so far. LSC precession happens only in the RBC case (Khalilov et al. 2018). Only one LSC reversal was captured in a seven-hour-long RBC experiment in liquid sodium (Mamykin et al. 2018). Further investigations regarding LSC cessations and reversals need to be carried out in the future. 


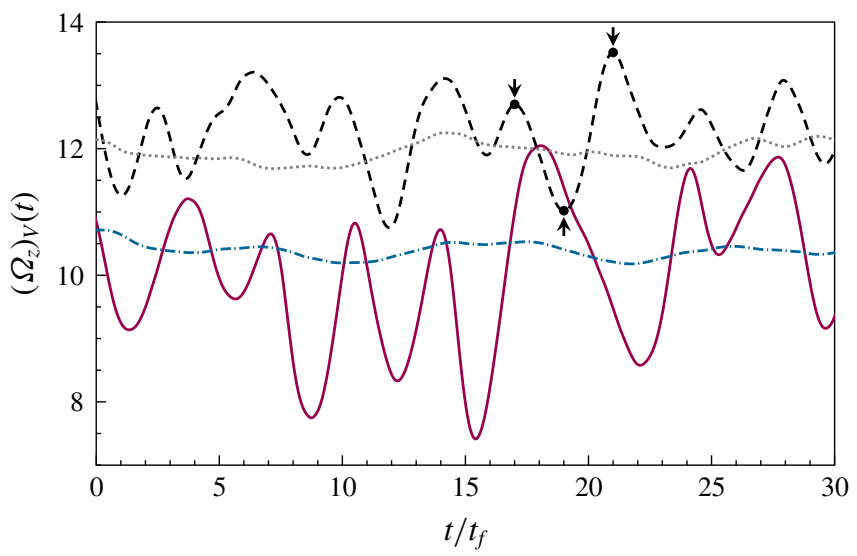

FIGURE 10. Time dependences of the volume-averaged component of the heat-flux vector along the cylinder axis, $\left\langle\Omega_{z}\right\rangle_{V}$, as obtained in the DNS for $\operatorname{Ra}=1.67 \times 10^{7}, \operatorname{Pr}=0.0094$ and four different inclination angles $\beta=0^{\circ}$ (solid line), $\beta=36^{\circ}$ (dash line), $\beta=72^{\circ}$ (dotted line) and $\beta=90^{\circ}$ (dash-dotted line). Time is normalised with $t_{f}=R(\alpha g R \Delta)^{-1 / 2}$. The arrows indicate the dimensionless times, which are marked in figure 12 with vertical lines and corresponding 3-dimensional snapshots are shown in figure 13.

In figure 10, the time evolution of the volume-averaged components of the heat-flux vector parallel to the cylinder axis, $\left\langle\Omega_{z}\right\rangle_{V}$ are presented, as they are obtained in the DNS for $R a=1.67 \times 10^{7}, \operatorname{Pr}=0.0094$ and four different inclination angles between $\beta=0^{\circ}$ (RBC) and $\beta=90^{\circ}$ (VC). Obviously, in the RBC case, the fluctuations of the heat flux around its mean value are extreme and reach up to $\pm 44 \%$ of $\left\langle\Omega_{z}\right\rangle$. The strength of the fluctuations gradually decreases with growing inclination angle $\beta$ and amount only to $\pm 3 \%$ of $\left\langle\Omega_{z}\right\rangle$ in the $\mathrm{VC}$ case. In figure 10 one can see that for the inclination angles $\beta=36^{\circ}$ and $\beta=72^{\circ}$, the mean heat transport is stronger than in the $\mathrm{RBC}$ or VC cases. This supports a general tendency that in small-Pr fluids the heat transport becomes more efficient, when the convection cell is tilted. Figure $5(a)$ and table 3 provide a more detailed evidence of this fact, based on our measurements and numerical simulations.

\subsubsection{The twisting mode}

In figure 11 we present the dynamics of the LSC twisting mode. There, the temporal evolution of the phases of the LSC in circle 1 (closer to the heated plate) and in circle 5 (closer to the cold plate) are presented for different inclinations angles $\beta$ of the convection cell filled with liquid sodium, as obtained in our DNS and measurements.

The main evidence for the existence of the twisting mode is the visible strong anticorrelation of the phases $\theta_{1}(t)$ and $\theta_{5}(t)$, which are measured via the probes at circles 1 and 5, respectively. It is present in the $\mathrm{RBC}$ case (figure $11 a, b$ ) and for the inclination angles $\beta=20^{\circ}$ (figure $11 c$ ) and $\beta=36^{\circ}$ (figure $11 d$ ). The measurements and DNS at the inclination angles $\beta \geqslant 40^{\circ}$ (figure 11e) show that, with increasing $\beta$, the above anti-correlation vanishes. At large inclination angles, there is no visible anti-correlation of the phases $\theta_{1}(t)$ and $\theta_{5}(t)$ and one can conclude that the twisting movement of the LSC is not present any more (figure $11 f, g, h$ ).

The dynamics of the twisting mode of the LSC can be further studied with the Fourier analysis. Thus, from the DNS data $\left(\operatorname{Ra}=1.67 \times 10^{7}, \operatorname{Pr}=0.0094\right)$ we obtain 


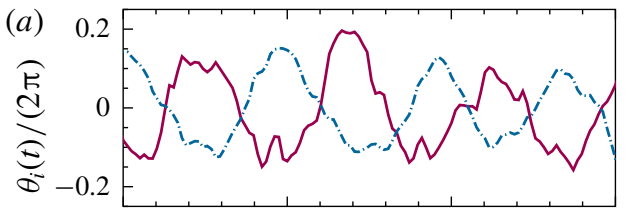

(b)
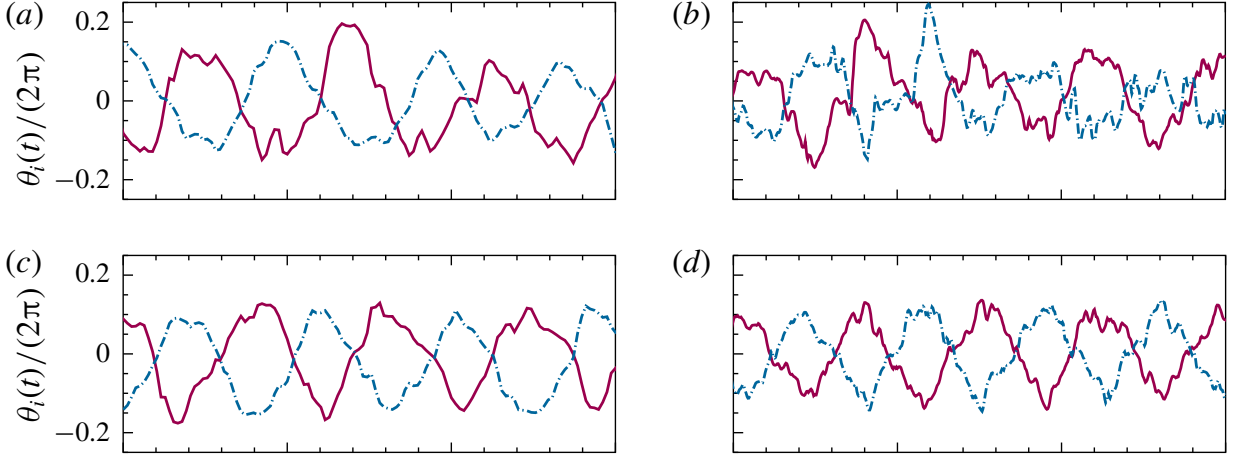

(d)
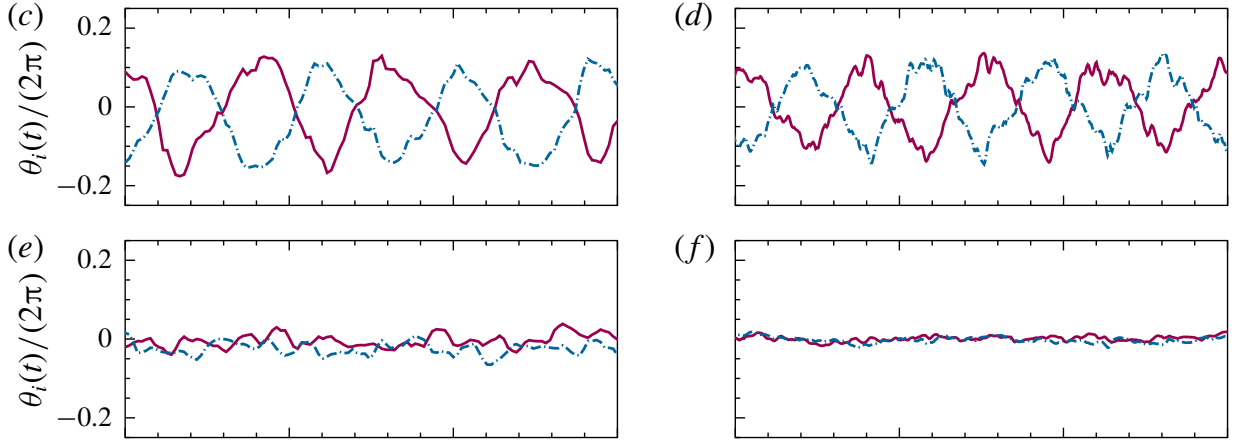

$(f)$
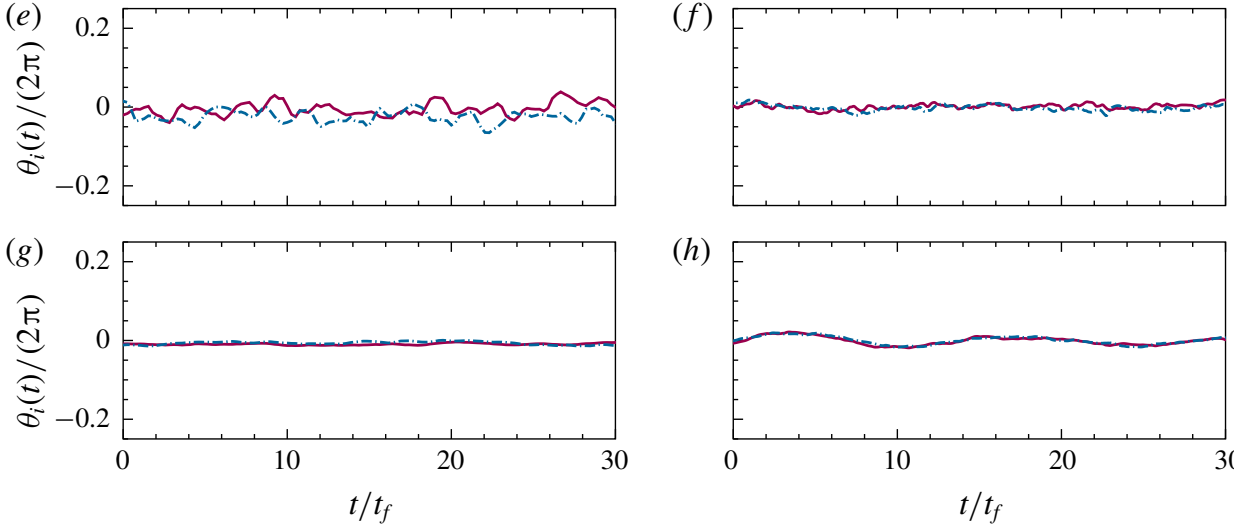

(h)

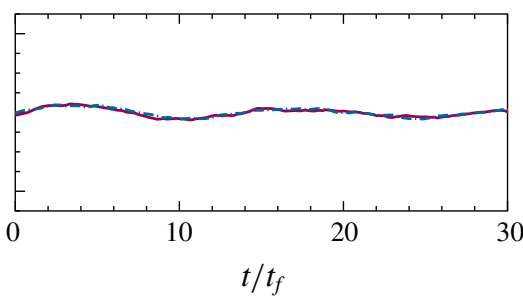

FIGURE 11. Temporal evolution of the phase $\theta_{1}$ in circle 1 (solid lines) and of the phase $\theta_{5}$ in circle 5 (dash-dotted line) of the convection cell (see locations of the circles in figure 2), as obtained in the experiments for $\operatorname{Ra}=1.42 \times 10^{7}, \operatorname{Pr} \approx 0.0093(a, c, e, g)$ and in the DNS for $\operatorname{Ra}=1.67 \times 10^{7}, \operatorname{Pr}=0.0094(b, d, f, h)$ for the cell inclination angles $\beta=0^{\circ}$ $(a, b), \beta=20^{\circ}(c), \beta=36^{\circ}(d), \beta=40^{\circ}(e), \beta=72^{\circ}(f)$ and $\beta=90^{\circ}(g, h)$.

that the period duration $T_{s}(\beta)$ equals $T_{s}\left(0^{\circ}\right)=8.8 t_{f}$ for the RBC case and is equal to $T_{s}\left(36^{\circ}\right)=7.4 t_{f}$ for the inclination angle $\beta=36^{\circ}$. The experimental data give $T_{s}\left(0^{\circ}\right) \approx$ $9.2 t_{f}$ for $\mathrm{RBC}$ and $T_{s}\left(20^{\circ}\right) \approx 8.7 t_{f}$ for $\beta=20^{\circ}$. Note that the frequency $\omega$ of the LSC twisting is approximately proportional to the Reynolds number $\omega \cdot t_{\kappa} \sim \operatorname{Re}$ (Cioni et al. 1997). Therefore, the slightly larger period durations in the experiments compared to those in the DNS are consistent with slightly lower Reynolds numbers and Rayleigh numbers there.

We also compare the twisting frequencies obtained in our DNS with results by Schumacher et al. (2016), Xi et al. (2009), Xie, Wei \& Xia (2013) and Funfschilling $\&$ Ahlers (2004). Note that we converted all numbers to free-fall time units $\left(t_{f}=\right.$ $R(\alpha g R \Delta)^{-1 / 2}$ ). For low Prandtl numbers, the numerical results by Schumacher et al. (2016) give a period of $4.6 t_{f}$ for $\operatorname{Pr}=0.021$ and $R a=10^{7}$. At higher Prandtl numbers the period is much longer. For water it is approximately $30 t_{f}$ at $\operatorname{Pr}=5.3$ and $\operatorname{Ra}=$ $5 \times 10^{9}$ (Xi et al. 2009). For even lager Prandtl number, $\operatorname{Pr}=19.4$, Xie et al. (2013) found $T_{s} \approx 85 t_{f}$ at $R a=2 \times 10^{11}$. The twisting frequency is lower with increasing $\operatorname{Pr}$ at high Prandtl numbers $(\operatorname{Pr}>1)$. However, the twisting frequency is slightly lower at $\operatorname{Pr}=0.0094$ compared to $\operatorname{Pr}=0.021$ for similar Rayleigh number. 

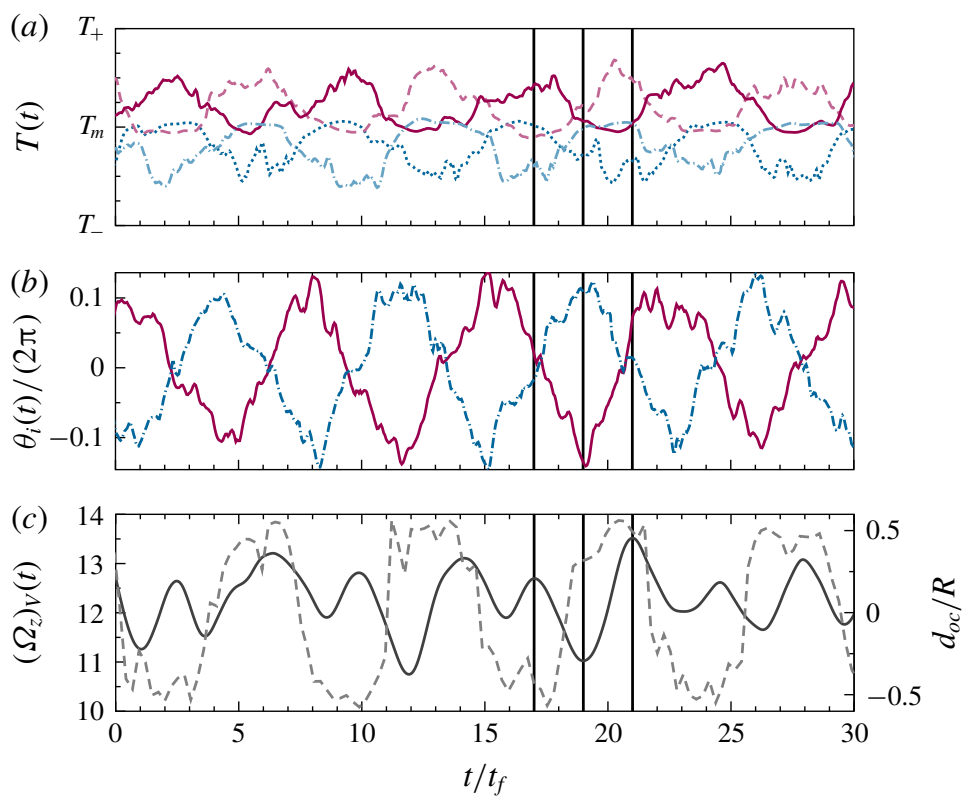

FIgURE 12. Temporal evolution of different quantities obtained in the DNS for $R a=$ $1.67 \times 10^{7}, \operatorname{Pr}=0.0094$ and the cell inclination angle $\beta=36^{\circ}:(a)$ the temperature $T$ at the probes B3 (solid line), D3 (dash-dotted line), F3 (dotted line) and H3 (dash line); (b) the phase $\theta_{1}$ in circle 1 (solid line) and the phase $\theta_{5}$ in circle 5 (dash-dotted line) and $(c)$ the volume-averaged component of the heat-flux vector along the cylinder axis, $\left\langle\Omega_{z}\right\rangle_{V}$ (solid line) and the off-centre distance, $d_{o c}$ (dashed line). The three vertical lines mark the times at which the snapshots in figure 13 are taken. The phases $\theta_{1}(t)$ and $\theta_{5}(t)$ in $(b)$ have a period of $T_{\theta}=(7.4 \pm 0.2) t_{f}$, which is determined by the Fourier analysis.

\subsubsection{The sloshing mode}

Besides the twisting mode there is another mode present in the flow. This is the sloshing mode; it brings together the hot and cold streams of the LSC. Both the twisting and the sloshing mode are observed simultaneously, and the full LSC dynamics is determined by a combination of both modes. To quantify the sloshing motion, we calculate the off-centre distance (2.19) and plot it over time (figure 12c) for the example case (DNS, $R a=1.67 \times 10^{7}, P r=0.0094, \beta=36^{\circ}$ ). The sloshing mode has the same frequency as the twisting mode (correlation of both signals is $>0.8$ ), but it shows a phase difference of approximately $\frac{1}{4}$-period. From the comparison of $d_{o c}(t)$ and $\left\langle\Omega_{z}\right\rangle_{V}(t)$ we learn that, first the hot and cold streams are brought close to each other, and then, with a short delay, the heat transport increases.

The sloshing mode is coherent through the entire height of the cylinder, quantified by a correlation of at least 0.8 between the sloshing signal $\left(d_{o c}\right)$ at mid-height circle 3 and the lower/higher circles (1,2, 4 and 5). The correlation of circles 1 and 5 is approximately 0.75 .

Let us investigate the IC flow in liquid sodium for the inclination angle $\beta=36^{\circ}$, as in figure $11(d)$, where a very strong LSC twisting is observed. In figure 12 we analyse this flow in more detail. Figure 12(a) presents the evolution of the temperature in time, which is measured by the probes B3, D3, F3 and H3 that are placed in the central circle 3 . 
(a)

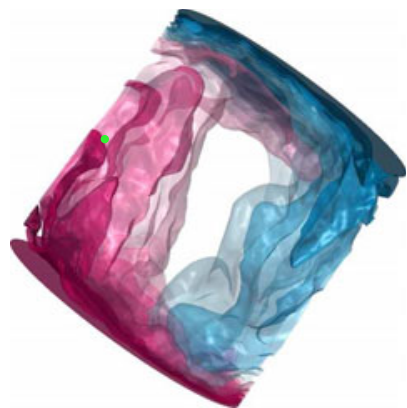

(d)

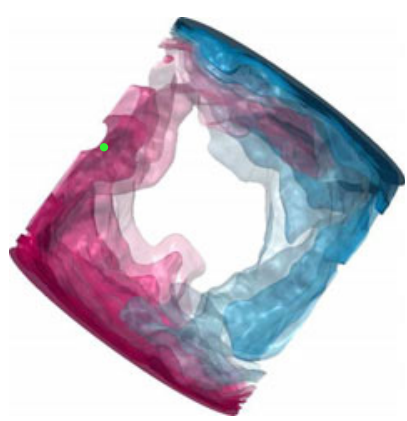

$(g)$

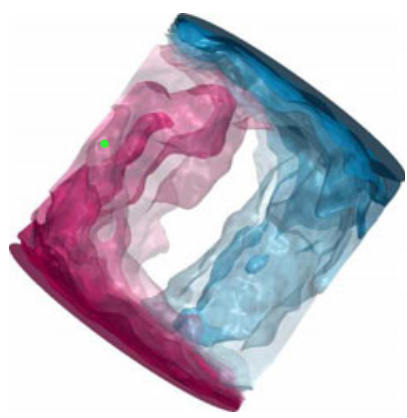

(b)

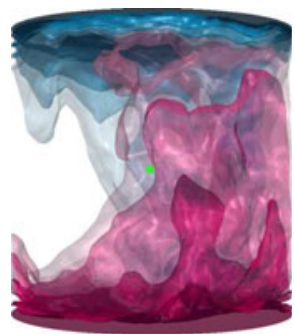

(e)

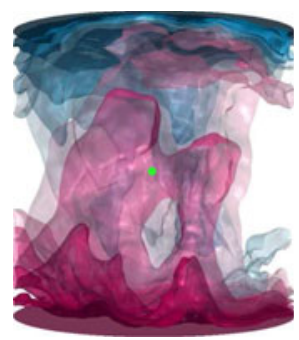

(h)

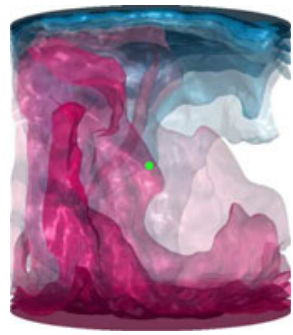

(c)

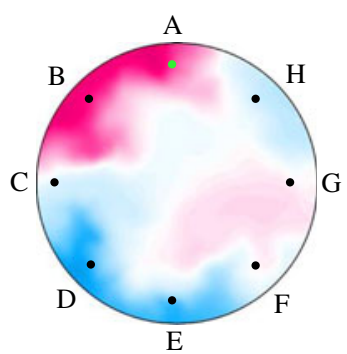

(f)

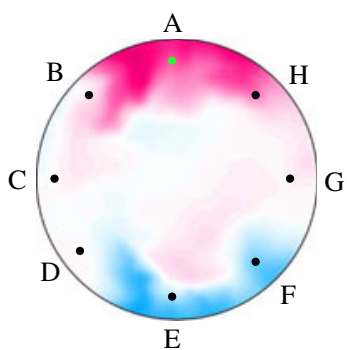

(i)

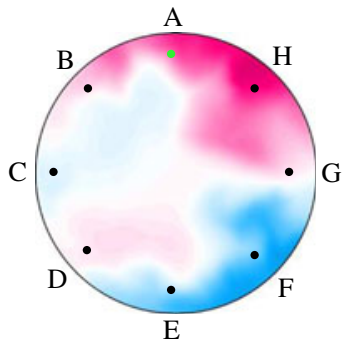

FIGURE 13. Three-dimensional side views $(a, d, g)$ and views orthogonal to the side views and to the cylinder axis $(b, e, h)$ of the temperature isosurfaces and the corresponding horizontal slices at the mid-height of the instantaneous temperature fields as seen from the cold plate $(c, f, i)$, which are obtained in the DNS for $R a=1.67 \times 10^{7}, \operatorname{Pr}=0.0094$ and the cell inclination angle $\beta=36^{\circ}$ at the times $(a, b, c) t=17 t_{f},(d, e, f) t=19 t_{f},(g, h, i) t=21 t_{f}$. The dot (green online) marks the location A3. The snapshots presented here correspond to the times marked in figure 12 with the vertical lines.

One can see that the temperature dependencies on time at the locations B3 and F3 are synchronous. So are the temperature dependencies on time at the locations H3 and D3. The temperatures at the locations B3 and D3 are anti-correlated. So are the temperatures at the locations H3 and F3. Thus, when at the location B3 the fluid is extremely hot, the lowest temperature is obtained near D3, which is located only $90^{\circ}$ azimuthally below B3. Analogously, when the fluid is hot at the location H3, its lowest temperature is obtained near the location F3, which is $90^{\circ}$ below $\mathrm{H} 3$ (see also figure 13). These events happen at the times $t / t_{f}=17$ and $t / t_{f}=21$ in figure 12 , respectively. Thus, at the times $t / t_{f}=17$ and $t / t_{f}=21$ a big hot and a big cold 
plume approach each other very closely. This sloshing movement happens periodically, alternately, near one side of the sidewall, then on the opposite side.

Figure $12(b)$ presents the evolution in time of the LSC phase $\theta_{1}$ in circle 1 (close to the heated plate) and of the phase $\theta_{5}$ in circle 5 (close to the cold plate).

These phases are anti-correlated, and the twisting of the LSC near the plates is maximal (at $\left.t / t_{f}=19\right)$ when the hot and the cold streams of the LSC in the central cross-section are located near the opposite sides of the cylinder sidewall.

In figure $12(c)$ the temporal evolution of the volume-averaged heat-flux vector parallel to the cylinder axis, $\left\langle\Omega_{z}\right\rangle_{V}$, is presented. Again, a very strong relationship with the LSC twisting and sloshing is observed. The maximal values of $\left\langle\Omega_{z}\right\rangle_{V}$ are obtained when the hot and cold LSC streams meet, thanks to sloshing, while the minimum value is obtained at the time periods when the LSC is strongly twisted.

In figure 13, the above described process, namely, the azimuthal movement of the hot and cold batches of fluid in the form of an oscillatory motion against each other, is illustrated with three-dimensional side views in two perpendicular directions. Additionally, the corresponding horizontal cross-sections of the instantaneous temperature fields at the mid-height of the cylinder are presented there. In the supplementary movies to this paper, available at https://doi.org/10.1017/jfm.2019.935, the described dynamics of the LSC can be observed in detail.

\subsubsection{Statistics of the large-scale circulation}

In figure 14(a) the standard deviations of the phases $\theta_{i}$ in the circles $i=1,3$ and 5 are presented, while figure 14(b) shows the corresponding time-averaged strengths of the LSC, $\left\langle\delta_{i}\right\rangle_{t}$, as they are obtained in the liquid-sodium measurements and DNS. The measurements show that the standard deviations of the phases $\theta_{1}$ (near the heated plate) and $\theta_{5}$ (near the cooled plate) are relatively large for small inclination angles, while being small for large inclination angles. There exist almost immediate drops of $\theta_{1}(\beta)$ and $\theta_{5}(\beta)$ that happen between $\beta=20^{\circ}$ and $\beta=40^{\circ}$, which indicate a sharp transition between the twisting and sloshing modes of the LSC and the usual mode of the LSC, when it is not twisted and located basically in the central vertical cross-section along the axis of the cylindrical sample. The standard deviations of $\theta_{1}$, $\theta_{3}$ and $\theta_{5}$, obtained in the DNS, show generally a similar behaviour as those measured in the experiments, but due to only a few considered inclination angles in the DNS, it is impossible to resolve the sudden drop which is observed in the measurements. Also one should notice that the data in figure 14 are very sensitive to the time of statistical averaging, which is extremely short in the DNS compared to the experiment.

The results for the time-averaged strengths of the LSC, $\left\langle\delta_{i}\right\rangle_{t}$, obtained in the measurements and DNS (figure 14b) show similar trends. In the RBC case $\left(\beta=0^{\circ}\right)$, the LSC strength is small and grows smoothly with the inclination angle $\beta$. However, the mean strength of the LSC in the measurements is approximately $0.02 \Delta$, i.e. $2 \%$ of the temperature difference, weaker that the one in the liquid-sodium DNS. This is consistent with the lower Nusselt number, as well as the lower Rayleigh number of the experiments. Surprisingly, the liquid-sodium DNS data show a similar strength of the LSC as the data from the auxiliary DNS for the same $R a P r$.

Evaluating the LSC phase angle $\theta_{i}$ and strength $\delta_{i}$ using the cos method (Cioni et al. 1997) by fitting only eight probes results in an uncertainty of approximately $8.6^{\circ}$ and $12.2 \%$, respectively. In table 5 we compare the mean deviation of the fit with a given number of probes $n$ compared to the case $n=512$. We find that the use of 32 probes is sufficient to reduce the uncertainty to below $1^{\circ}$ and $1 \%$, respectively. However, using only eight probes in the experiment is acceptable, especially considering that every additional probe disturbs the flow. 

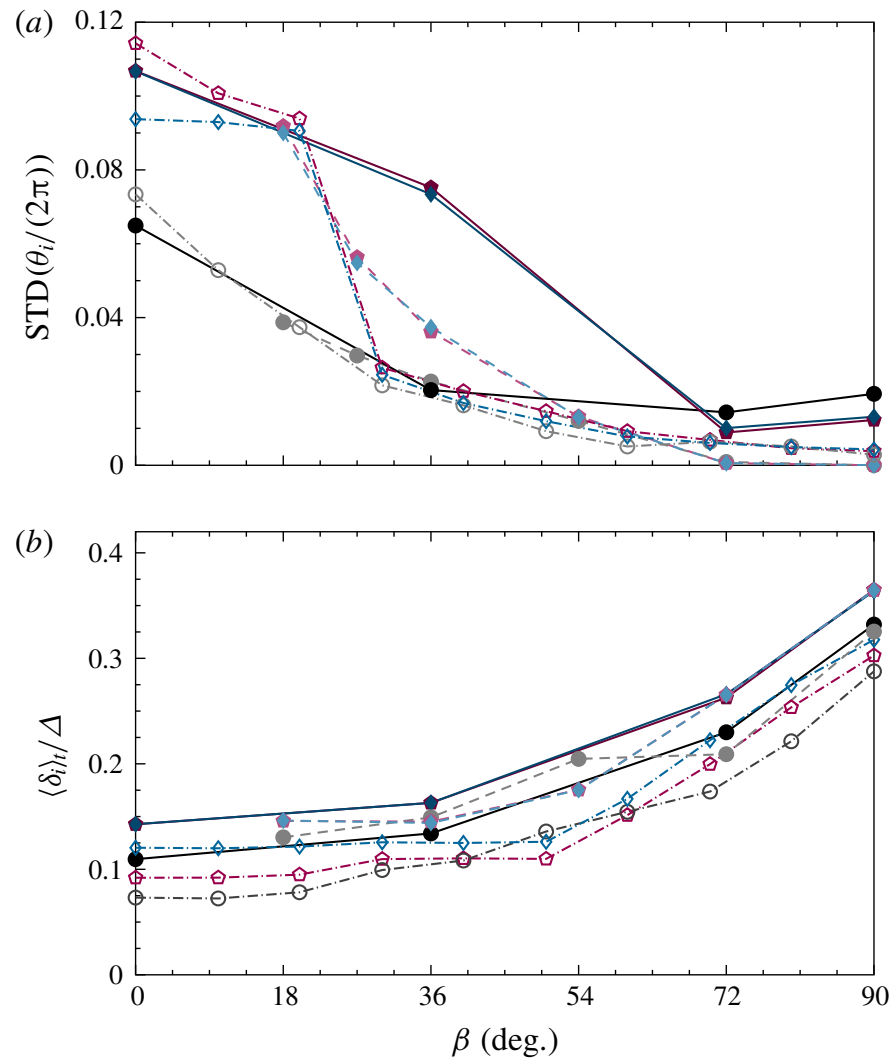

FIGURE 14. (a) Standard deviations of the phase angles $\theta_{i}$ in radians normalised by $2 \pi$ and $(b)$ the time-averaged strengths of the large-scale circulation, $\left\langle\delta_{i}\right\rangle_{t}$, as obtained at the probe circle 1 (pink colour symbols and lines), circle 3 (grey colour) and circle 5 (blue colour) in the experiments (dash-dotted lines), the DNS for $\operatorname{Ra}=1.67 \times 10^{7}, \operatorname{Pr}=0.0094$ (solid lines) and DNS for $\operatorname{Ra}=10^{6}, \operatorname{Pr}=0.094$ (dash lines).

$\begin{array}{rlc}n & \theta_{\text {err }}(\mathrm{deg} .) & \delta_{\text {err }}(\%) \\ 8 & 8.6 & 12.2 \\ 16 & 2.6 & 3.6 \\ 32 & 0.6 & 0.8 \\ 64 & 0.07 & 0.1\end{array}$

TABLE 5. Uncertainties $\theta_{\text {err }}=\left|\theta^{(n)}-\theta^{(512)}\right|$ and $\delta_{\text {err }}=\mid \delta^{(n)} / \delta^{(512)}-1$.| that are caused by the limited number of probes $n$ while using the cos method to determine the phase $\theta_{i}$ and strength $\delta_{i}$ of the LSC. All values are averaged over 5 circles and 400 snapshots of the DNS data for $R a=1.67 \times 10^{7}, \operatorname{Pr}=0.0094, \beta=0^{\circ}$.

\section{Conclusions}

In our complementary and cross-validating experimental and numerical studies, we have investigated inclined turbulent thermal convection in liquid sodium $(\mathrm{Pr} \approx$ 0.009) in a cylindrical container of aspect ratio one. The results of the conducted 
measurements, DNS and LES demonstrated a good quantitative agreement, and the uncertainty margin has been extensively discussed.

For the limiting cases of inclined convection, which are Rayleigh-Bénard convection (with the cell inclination angle $\beta=0^{\circ}$ ) and vertical convection $\left(\beta=90^{\circ}\right)$, we have experimentally studied the scaling relations of the mean heat flux (Nusselt number) with Rayleigh number, for $R a$ around $10^{7}$. The scaling exponents were found to be approximately 0.22 in both cases, but the absolute values of $N u$ are found to be larger in $\mathrm{VC}$, compared to those in RBC. At the considered Rayleigh number of approximately $1.5 \times 10^{7}$, any inclination of the RBC cell generally leads to an increase of the mean heat flux. The maximal $N u$ is obtained, however, for a certain intermediate value of $\beta$ and may increase up to $29 \%$ (compared to the RBC case) for liquid sodium at the considered Rayleigh number.

It was proved, in particular, that the use of the cross-correlation time of the neighbouring temperature probes is a reliable tool to evaluate velocities during temperature measurements in liquid sodium.

We showed (cf. table 5) that, using the cos method (2.18) to determine the phase $\theta_{i}$ and strength $\delta_{i}$ of the LSC with only 8 probes, one obtains reliable results with an uncertainty of approximately $8.6^{\circ}$ and $\pm 12.2 \%$, respectively.

Furthermore, we have found that for small Prandtl numbers there exists a similarity of IC flows of the same $\operatorname{RaPr}$, due to the similar ratio, $t_{\kappa} / t_{f} \sim \sqrt{\operatorname{RaPr}}$, between the thermal diffusion time scale, $t_{\kappa}$, and the free-fall time scale, $t_{f}$. Since in small- $P r$ convective flows, the viscous diffusion time scale, $t_{v}$, is much larger than $t_{\kappa}$, the value of $R a P r$ determines basically the mean temperature and heat-flux distributions. The Nusselt numbers and the relative Reynolds numbers of the liquid-sodium DNS and auxiliary DNS at similar $\mathrm{RaPr}$ are similar for any inclination of the convection cell with respect to the gravity vector. This property can be very useful for the investigation of, e.g., the scaling relations of $N u$ and $R e$ with $R a$ and $\operatorname{Pr}$ or of the mean temperature or heat-flux distributions, at extremely high $R a$ and/or extremely small $\mathrm{Pr}$.

For small inclination angles, the large-scale circulation exhibits complex dynamics, with twisting and sloshing modes and these modes strongly influence the instantaneous heat transport in the system ( $\pm 44 \%$ of the mean heat transport). In a strongly twisted LSC, the volume-averaged vertical heat flux is minimal, and it is maximal, shortly after the LSC sloshing mode brought together the hot and cold streams of the LSC. Note that both modes are present in the flow simultaneously. Figures 12 and 13 and supplementary movies illustrate the studied LSC dynamics. Additional investigations will be needed to study the even more complex behaviour of the LSC in IC of low- $\mathrm{Pr}$ fluids in elongated containers with $L \gg D$.

\section{Acknowledgements}

This work is supported by the Priority Programme SPP 1881 Turbulent Superstructures of the Deutsche Forschungsgemeinschaft (DFG) under the grant Sh405/7. O.S. also thanks the DFG for the support under the grant Sh405/4 Heisenberg fellowship. The authors acknowledge the Leibniz Supercomputing Centre (LRZ) for providing computing time and the Institute of Continuous Media Mechanics (ICMM UB RAS) for providing resources of the Triton supercomputer.

\section{Supplementary movies}

Supplementary movies are available at https://doi.org/10.1017/jfm.2019.935. 


\section{REFERENCES}

Ahlers, G., Brown, E., Araujo, F. F., Funfschilling, D., Grossmann, S. \& Lohse, D. $2006 a$ Non-Oberbeck-Boussinesq effects in strongly turbulent Rayleigh-Bénard convection. J. Fluid Mech. 569, 409-446.

Ahlers, G., Brown, E. \& Nikolaenko, A. $2006 b$ The search for slow transients, and the effect of imperfect vertical alignment, in turbulent Rayleigh-Bénard convection. J. Fluid Mech. 557, 347-367.

Ahlers, G., Grossmann, S. \& Lohse, D. 2009 Heat transfer and large scale dynamics in turbulent Rayleigh-Bénard convection. Rev. Mod. Phys. 81, 503-537.

BAI, K., JI, D. \& BROWN, E. 2016 Ability of a low-dimensional model to predict geometry-dependent dynamics of large-scale coherent structures in turbulence. Phys. Rev. E 93, 023117.

Bailon-Cuba, J., Shishinan, O., Wagner, C. \& Schumacher, J. 2012 Low-dimensional model of turbulent mixed convection in a complex domain. Phys. Fluids 24, 107101.

Belyaev, I. A., Genin, L. G., Listratov, Y. I., Melnikov, I. A., Sviridov, V. G., Sviridov, E. V., Ivochioin, Y. P., Razuvanov, N. G. \& ShPansky, Y. S. 2013 Specific features of liquid metal heat transfer in a tokamak reactor. Magnetohydrodynamics 49, 177-190.

Blasius, H. 1908 Grenzschichten in Flüssigkeiten mit kleiner Reibung. Z. Math. Phys. 56, 1-37.

Bodenschatz, E., Pesch, W. \& Ahlers, G. 2000 Recent developments in Rayleigh-Bénard convection. Annu. Rev. Fluid Mech. 32, 709-778.

Brown, E. \& Ahlers, G. 2006 Rotations and cessations of the large-scale circulation in turbulent Rayleigh-Bénard convection. J. Fluid Mech. 568, 351-386.

Brown, E. \& AHLERs, G. 2009 The origin of oscillations of the large-scale circulation of turbulent Rayleigh-Bénard convection. J. Fluid Mech. 638, 383-400.

Chen, F., Huai, X., CaI, J., LI, X.\& MenG, R. 2013 Investigation on the applicability of turbulentPrandtl-number models for liquid lead-bismuth eutectic. Nucl. Engng Des. 257, 128-133.

Chillà, F., Rastello, M., Chaumat, S. \& Castaing, B. 2004 Long relaxation times and tilt sensitivity in Rayleigh-Bénard turbulence. Eur. Phys. J. B 40 (2), 223-227.

Chillà, F. \& Schumacher, J. 2012 New perspectives in turbulent Rayleigh-Bénard convection. Eur. Phys. J. E 35, 58.

Ching, E. S. C., Dung, O.-Y. \& Shishkina, O. 2017 Fluctuating thermal boundary layers and heat transfer in turbulent Rayleigh-Bénard convection. J. Stat. Phys. 167, 626-635.

Chong, K. L., Huang, S.-D., Kaczorowski, M. \& XIA, K.-Q. 2015 Condensation of coherent structures in turbulent flows. Phys. Rev. Lett. 115, 264503.

Chong, K. L., Wagner, S., Kaczorowski, M., Shishina, O. \& Xia, K.-Q. 2018 Effect of Prandtl number on heat transport enhancement in Rayleigh-Bénard convection under geometrical confinement. Phys. Rev. Fluids 3, 013501.

Chong, K. L. \& XIA, K.-Q. 2016 Exploring the severely confined regime in Rayleigh-Bénard convection. J. Fluid Mech. 805, R4.

Churchill, S. W. \& CHU, H. H. S. 1975 Correlating equations for laminar and turbulent free convection from a vertical plate. Intl J. Heat Mass Transfer 18, 1323-1329.

Ciliberto, S., Cioni, S. \& Laroche, C. 1996 Large-scale flow properties of turbulent thermal convection. Phys. Rev. E 54, R5901-R5904.

Cioni, S., Ciliberto, S. \& Sommeria, J. 1997 Strongly turbulent Rayleigh-Bénard convection in mercury: comparison with results at moderate Prandtl number. J. Fluid Mech. 335, 111-140.

Daniels, K. E., Wiener, R. J. \& Bodenschatz, E. 2003 Localized transverse bursts in inclined layer convection. Phys. Rev. Lett. 91, 114501.

DeARdorfF, J. W. 1970 A numerical study of three-dimensional turbulent channel flow at large Reynolds numbers. J. Fluid Mech. 41, 453-480.

Ferziger, J. H. \& Perić, M. 2002 Computational Methods for Fluid Dynamics, 3rd edn. Springer.

Fletcher, R. 1976 Conjugate gradient methods for indefinite systems. In Numerical Analysis, Lecture Notes in Mathematics, vol. 506. Springer.

Frick, P., Khalilov, R., Kolesnichenko, I., Mamykin, A., Pakholkov, V., Pavlinov, A. \& RogozhKin, S. A. 2015 Turbulent convective heat transfer in a long cylinder with liquid sodium. Eur. Phys. Lett. 109, 14002. 
Fuji, T., TAkeuchi, M., Fujis, M., Suzaki, K. \& Uehar, H. 1970 Experiments on naturalconvection heat transfer from the outer surface of a vertical cylinder to liquids. Intl J. Heat Mass Transfer 13, 753-770.

Funfschilling, D. \& Ahlers, G. 2004 Plume motion and large-scale dynamics in a cylindrical Rayleigh-Bénard cell. Phys. Rev. Lett. 92, 194502.

Funfschilling, D., Brown, E. \& Ahlers, G. 2008 Torsional oscillations of the large-scale circulation in turbulent Rayleigh-Bénard convection. J. Fluid Mech. 607, 119-139.

George, W. K. \& CAPP, S. P. 1979 A theory for natural convection turbulent boundary layers next to heated vertical surfaces. Intl J. Heat Mass Transfer 22, 813-826.

Glazier, J. A., Segawa, T., NAert, A. \& Sano, M. 1999 Evidence against 'ultrahard' thermal turbulence at very high Rayleigh numbers. Nature 398, 307-310.

Grossmann, S. \& Lohse, D. 2000 Scaling in thermal convection: a unifying theory. J. Fluid Mech. 407, 27-56.

Grossmann, S. \& Lohse, D. 2001 Thermal convection for large Prandtl numbers. Phys. Rev. Lett. 86, 3316-3319.

Grossmann, S. \& Lohse, D. 2011 Multiple scaling in the ultimate regime of thermal convection. Phys. Fluids 23, 045108.

Guo, S.-X., Zhou, S.-Q., Cen, X.-R., Qu, L., Lu, Y.-Z., Sun, L. \& Shang, X.-D. 2015 The effect of cell tilting on turbulent thermal convection in a rectangular cell. J. Fluid Mech. 762, 273-287.

Hanasoge, S., Gizon, L. \& Sreenivasan, K. R. 2016 Seismic sounding of convection in the sun. Annu. Rev. Fluid Mech. 48, 191-217.

He, X., Bodenschatz, E. \& Ahlers, G. 2016 Azimuthal diffusion of the large-scale-circulation plane, and absence of significant non-Boussinesq effects, in turbulent convection near the ultimate-state transition. J. Fluid Mech. 791, R3.

Heinzel, A., Hering, W., Konys, J., Marocco, L., Litfin, K., Mueller, G., Pacio, J., Schroer, C., Stieglitz, R., Stoppel, L. et al. 2017 Liquid metals as efficient hightemperature heat-transport fluids. Energy Technol. 5, 1026-1036.

Horanyi, S., Krebs, L. \& Müller, U. 1999 Turbulent Rayleigh-Bénard convection low Prandtlnumber fluids. Intl J. Heat Mass Transfer 42, 3983-4003.

HoRN, S. \& SCHMID, P. J. 2017 Prograde, retrograde, and oscillatory modes in rotating RayleighBénard convection. J. Fluid Mech. 831, 182-211.

Huang, S.-D., Kaczorowski, M., Ni, R. \& XIA, K.-Q. 2013 Confinement induced heat-transport enhancement in turbulent thermal convection. Phys. Rev. Lett. 111, 104501.

IssA, R. 1986 Solution of the implicitly discretised fluid flow equations by operator-splitting. J. Comput. Phys. 62, 40-65.

Khalilov, R., Kolesnichenko, I., Pavlinov, A., Mamykin, A., Shestakov, A. \& Frick, P. 2018 Thermal convection of liquid sodium in inclined cylinders. Phys. Rev. Fluids 3, 043503.

King, E. M. \& Aurnou, J. M. 2013 Turbulent convection in liquid metal with and without rotation. Proc. Natl Acad. Sci. USA 110, 6688-6693.

KIS, P. \& HeRwig, H. 2012 The near wall physics and wall functions for turbulent natural convection. Intl J. Heat Mass Transfer 55, 2625-2635.

Kolesnichenko, I., Khalilov, R., Teimurazov, A. \& Frick, P. 2017 On boundary conditions in liquid sodium convective experiments. J. Phys.: Conf. Ser. 891, 012075.

Kolesnichenko, I. V., Mamykin, A. D., Pavlinov, A. M., Pakholkov, V. V., Rogozhkin, S. A., Frick, P. G., Khalilov, R. I. \& ShepeleV, S. F. 2015 Experimental study on free convection of sodium in a long cylinder. Thermal Engng 62, 414-422.

Kooij, G. L., Botchev, M. A., Frederix, E. M. A., Geurts, B. J., Horn, S., Lohse, D., VAn der Poel, E. P., Shishinina, O., Stevens, R. J. A. M. \& Verzicco, R. 2018 Comparison of computational codes for direct numerical simulations of turbulent RayleighBénard convection. Comput. Fluids 166, 1-8.

Kraichnan, R. 1962 Turbulent thermal convection at arbitrary Prandtl number. Phys. Fluids 5, 1374-1389. 
Landau, L. D. \& Lifshitz, E. M. 1987 Fluid Mechanics, 2nd edn. Course of Theoretical Physics, vol. 6. Butterworth Heinemann.

LANGebach, R. \& HABerstroh, C. 2014 Natural convection in inclined pipes - a new correlation for heat transfer estimations. In Advances in Cryogenic Engineering (ed. J. G. Weisend et al.), AIP Conf. Proc., vol. 1573, pp. 1504-1511.

Lohse, D. \& XIA, K.-Q. 2010 Small-scale properties of turbulent Rayleigh-Bénard convection. Annu. Rev. Fluid Mech. 42, 335-364.

LorenZ, H. H. 1934 Die Wärmeübertragung von einer ebenen, senkrechten Platte an öl bei natürlicher Konvektion. Zs. f. tech. Phys. 9 (15), 362-366.

Mamykin, A., Frick, P., Khalilov, R., Kolesnichenko, I., Pakholkov, V., Rogozhkin, S. \& VASILIEV, A. 2015 Turbulent convective heat transfer in an inclined tube with liquid sodium. Magnetohydrodynamics 51, 329-336.

Mamykin, A. D., Kolesnichenko, I. V., Pavlinov, A. M. \& Khalilov, R. I. 2018 Large scale circulation in turbulent Rayleigh-Bénard convection of liquid sodium in cylindrical cell. J. Phys.: Conf. Ser. 1128, 012019.

MANDRYKin, S. D. \& Teimurazov, A. S. 2019 Turbulent convection of liquid sodium in an inclined cylinder of unit aspect ratio. J. Appl. Mech. Tech. Phys. 60 (7) (in press).

Mishra, P. K., De, A. K., Verma, M. K. \& Eswaran, V. 2011 Dynamics of reorientations and reversals of large-scale flow in Rayleigh-Bénard convection. J. Fluid Mech. 668, 480-499.

NG, C. S., OoI, A., Lohse, D. \& Chung, D. 2015 Vertical natural convection: application of the unifying theory of thermal convection. J. Fluid Mech. 764, 349-361.

NG, C. S., Ool, A., Lohse, D. \& ChUnG, D. 2017 Changes in the boundary-layer structure at the edge of the ultimate regime in vertical natural convection. J. Fluid Mech. 825, 550-572.

NG, C. S., OoI, A., Lohse, D. \& ChUnG, D. 2018 Bulk scaling in wall-bounded and homogeneous vertical natural convection. J. Fluid Mech. 841, 825-850.

Onea, A., Hering, W., LuX, M. \& Stieglitz, R. 2017 a Numerical optimization of cold trap designs for the Karlsruhe Sodium Laboratory. Intl J. Heat Mass Transfer 113, 984-999.

Onea, A., Perez-Martin, S., Jaeger, W., Hering, W. \& Stieglitz, R. $2017 b$ Liquid Metals as Heat Transfer Fluids for Science and Technology, 1st edn. Advances in New Heat Transfer Fluids. From Numerical to Experimental Techniques. CRC Press.

PRANDTL, L. 1905 Über Flüssigkeitsbewegung bei sehr kleiner Reibung. In Verhandlungen des III. Int. Math. Kongr., Heidelberg, 1904, pp. 484-491. Teubner.

Riedinger, X., Tisserand, J.-C., Seychelles, F., Castaing, B. \& Chillá, F. 2013 Heat transport regimes in an inclined channel. Phys. Fluids 25, 015117.

Roche, P.-E., Gauthier, F., Kaiser, R. \& SAlort, J. 2010 On the triggering of the ultimate regime of convection. New J. Phys. 12 (8), 085014.

Saunders, O. A. 1939 Natural convection in liquids. Proc. R. Soc. Lond. A 172 (948), 55-71.

SCHeEl, J. D. \& SChumacher, J. 2016 Global and local statistics in turbulent convection at low Prandtl numbers. J. Fluid Mech. 802, 147-173.

Scheel, J. D. \& Schumacher, J. 2017 Predicting transition ranges to fully turbulent viscous boundary layers in low Prandtl number convection flows. Phys. Rev. Fluids 2, 123501.

Schlichting, H. \& Gersten, K. 2000 Boundary-Layer Theory, 8th edn. Springer.

Schmidt, E. \& BeckMAnN, W. 1930 Das Temperatur- und Geschwindigskeitsfeld vor einer Wärme abgebenden senkrechten Platte bei natürlicher Konvektion. Forsch. Ing.-Wes. (Techn. Mech. Thermodyn.) 1, 341-349, 391-406.

Schumacher, J., Bandaru, V., Pandey, A. \& Scheel, J. D. 2016 Transitional boundary layers in low-Prandtl-number convection. Phys. Rev. Fluids 1, 084402.

Shishkina, O. 2016 Momentum and heat transport scalings in laminar vertical convection. Phys. Rev. E 93, 051102(R).

Shishinina, O., Emran, M., Grossmann, S. \& Lohse, D. 2017 Scaling relations in large-Prandtlnumber natural thermal convection. Phys. Rev. Fluids 2, 103502.

Shishina, O., Grossmann, S. \& Lohse, D. $2016 a$ Heat and momentum transport scalings in horizontal convection. Geophys. Res. Lett. 43, 1219-1225. 
Shishrina, O. \& Horn, S. 2016 Thermal convection in inclined cylindrical containers. J. Fluid Mech. 790, R3.

Shishina, O., Stevens, R. J. A. M., Grossmann, S. \& Lohse, D. 2010 Boundary layer structure in turbulent thermal convection and its consequences for the required numerical resolution. New J. Phys. 12, 075022.

Shishinina, O., Weiss, S. \& Bodenschatz, E. $2016 b$ Conductive heat flux in measurements of the Nusselt number in turbulent Rayleigh-Bénard convection. Phys. Rev. Fluids 1, 062301(R).

SpIEgEL, E. A. 1962 Thermal turbulence at very small Prandtl number. J. Geophys. Res. 67, 3063-3070.

Stevens, R. J. A. M., Clercx, H. J. H. \& Lohse, D. 2011 Effect of plumes on measuring the large scale circulation in turbulent Rayleigh-Bénard convection. Phys. Fluids 23, 095110.

Stevens, R. J. A. M., van Der Poel, E. P., Grossmann, S. \& Lohse, D. 2013 The unifying theory of scaling in thermal convection: the updated prefactors. J. Fluid Mech. 730, 295-308.

Sun, C., XI, H.-D. \& XIA, K.-Q. 2005 Azimuthal symmetry, flow dynamics, and heat transport in turbulent thermal convection in a cylinder with an aspect ratio of 0.5. Phys. Rev. Lett. 95, 074502.

Takeshita, T., Segawa, T., Glazier, J. A. \& Sano, M. 1996 Thermal turbulence in mercury. Phys. Rev. Lett. 76, 1465-1468.

Teimurazov, A. \& Frick, P. 2017 Thermal convection of liquid metal in a long inclined cylinder. Phys. Rev. Fluids 2, 113501.

Teimurazov, A., Frick, P. \& Stefani, F. 2017 Thermal convection of liquid metal in the titanium reduction reactor. IOP Conf. Ser: Materials Sci. Engng 208, 012044.

Vasil'ev, A. Y., Kolesnichenko, I. V., Mamykin, A. D., Frick, P. G., Khalilov, R. I., RogozhKin, S. A. \& PAKHolkov, V. V. 2015 Turbulent convective heat transfer in an inclined tube filled with sodium. Tech. Phys. 60, 1305-1309.

Versteegh, T. A. M. \& Nieuwstadt, F. T. M. 1999 A direct numerical simulation of natural convection between two infinite vertical differentially heated walls scaling laws and wall functions. Intl J. Heat Mass Transfer 42, 3673-3693.

Vogt, T., Horn, S., Grannan, A. M. \& Aurnou, J. M. 2018 Jump rope vortex in liquid metal convection. Proc. Natl Acad. Sci. USA 115 (50), 12674-12679.

WAgner, S. \& ShishKinA, O. 2013 Aspect ratio dependency of Rayleigh-Bénard convection in box-shaped containers. Phys. Fluids 25, 085110.

WAGNER, S. \& SHISHKINA, O. 2015 Heat flux enhancement by regular surface roughness in turbulent thermal convection. J. Fluid Mech. 763, 109-135.

WAGNer, S., ShishKinA, O. \& WAGNeR, C. 2012 Boundary layers and wind in cylindrical RayleighBénard cells. J. Fluid Mech. 697, 336-366.

WEI, P. \& XIA, K.-Q. 2013 Viscous boundary layer properties in turbulent thermal convection in a cylindrical cell: the effect of cell tilting. J. Fluid Mech. 720, 140-168.

Weiss, S. \& AHLERS, G. 2013 Effect of tilting on turbulent convection: cylindrical samples with aspect ratio $\gamma=0.50$. J. Fluid Mech. 715, 314-334.

Weiss, S., He, X., Ahlers, G., Bodenschatz, E. \& Shishkina, O. 2018 Bulk temperature and heat transport in turbulent Rayleigh-Bénard convection of fluids with temperature-dependent properties. J. Fluid Mech. 851, 374-390.

Weller, H. G., TABor, G., JASAK, H. \& Fureby, C. 1998 A tensorial approach to computational continuum mechanics using object-oriented techniques. Comput. Phys. 12, 620-631.

XI, H.-D. \& XIA, K.-Q. 2007 Cessations and reversals of the large-scale circulation in turbulent thermal convection. Phys. Rev. E 76, 036301.

XI, H.-D. \& XIA, K.-Q. 2008 Flow mode transitions in turbulent thermal convection. Phys. Fluids 20, 055104.

XI, H.-D., Zhou, Q. \& XIA, K.-Q. 2006 Azimuthal motion of the mean wind in turbulent thermal convection. Phys. Rev. E 73, 056312.

XI, H.-D., Zhou, S.-Q., Zhou, Q., Chan, T. S. \& XIA, K.-Q. 2009 Origin of the temperature oscillations in turbulent thermal convection. Phys. Rev. Lett. 102, 044503. 
Xie, H. Q., Wei, P. \& XiA, K.-Q. 2013 Dynamics of the large-scale circulation in high-Prandtlnumber turbulent thermal convection. J. Fluid Mech. 717, 322-346.

YU, H., LI, N. \& ECKE, R. E. 2007 Scaling in laminar natural convection in laterally heated cavities: is turbulence essential in the classical scaling of heat transfer? Phys. Rev. E 76, 026303.

Zhilin, V. G., Sviridov, V. G., Razuvanov, N. G., Ivochkin, Y. P., Listratov, Y. I., SViridov, E. V. \& BelyaeV, I. A. 2009 Influence of secondary flows on the liquid metal heat transfer in a horizontal pipe with nonuniform heating in presence of a magnetic field. Therm. Process Engng 1, 199-203.

Zhou, Q., XI, H.-D., Zhou, S.-Q., Sun, C. \& XIA, K.-Q. 2009 Oscillations of the large-scale circulation in turbulent Rayleigh-Bénard convection: the sloshing mode and its relationship with the torsional mode. J. Fluid Mech. 630, 367-390.

Zhou, Q. \& XIA, K.-Q. 2010 Measured instantaneous viscous boundary layer in turbulent RayleighBénard convection. Phys. Rev. Lett. 104, 104301.

Zimin, V. D., FriK, P. G. \& Shaidurov, V. G. 1982 Turbulent convection in a cubic cell heated simultaneously on the side and the bottom. Fluid Dyn. 17, 286-290.

Zürner, T., Schindler, F., Vogt, T., Eckert, S. \& Schumacher, J. 2019 Combined measurement of velocity and temperature in liquid metal convection. J. Fluid Mech. 876, $1108-1128$.

ZWIRNER, L. \& SHISHKINA, O. 2018 Confined inclined thermal convection in low-Prandtl-number fluids. J. Fluid Mech. 850, 984-1008. 